\title{
GENERAL ANALYSIS OF TYPE I PLANETARY MIGRATION WITH STOCHASTIC PERTURBATIONS
}

\author{
Fred C. Adams ${ }^{1,2}$ and Anthony M. Bloch ${ }^{1,3}$ \\ ${ }^{1}$ Michigan Center for Theoretical Physics \\ Physics Department, University of Michigan, Ann Arbor, MI 48109 \\ ${ }^{2}$ Astronomy Department, University of Michigan, Ann Arbor, MI 48109 \\ ${ }^{3}$ Department of Mathematics, University of Michigan, Ann Arbor, MI 48109
}

\begin{abstract}
This paper presents a generalized treatment of Type I planetary migration in the presence of stochastic perturbations. In many planet-forming disks, the Type I migration mechanism, driven by asymmetric torques, acts on a short time scale and compromises planet formation. If the disk also supports MHD instabilities, however, the corresponding turbulent fluctuations produce additional stochastic torques that modify the steady inward migration scenario. This work studies the migration of planetary cores in the presence of stochastic fluctuations using complementary methods, including a Fokker-Planck approach and iterative maps. Stochastic torques have two main effects: [1] Through outward diffusion, a small fraction of the planetary cores can survive in the face of Type I inward migration. [2] For a given starting condition, the result of any particular realization of migration is uncertain, so that results must be described in terms of the distributions of outcomes. In addition to exploring different regimes of parameter space, this paper considers the effects of the outer disk boundary condition, varying initial conditions, and time-dependence of the torque parameters. For disks with finite radii, the fraction of surviving planets decreases exponentially with time. We find the survival fractions and decay rates for a range of disk models, and find the expected distribution of locations for surviving planets. For expected disk properties, the survival fraction lies in the range $0.01<p_{S}<0.1$.
\end{abstract}

Subject headings: MHD — planetary systems — planetary systems: formation — planets and satellites: formation — turbulence

\section{INTRODUCTION}

The past decade has led to tremendous progress in our understanding of extrasolar planets and the processes involved in planet formation. These advances include both observations, which now include the detection of nearly 300 planets outside our Solar System (see, e.g., Udry et al. 2007 for 
a recent review), along with a great deal of accompanying theoretical work. One surprise resulting from the observations is the finding that extrasolar planets display a much wider range of orbital configurations than was anticipated. Planets thus move (usually inward) from their birth sites, or while they are forming, in a process known as planet migration (e.g., see Papaloizou \& Terquem 2006 for a recent review).

The migration process is especially rapid when the planets have small masses, less than $~ 30$ $M_{\oplus}$, so they cannot clear gaps in the disks (Goldreich \& Tremaine 1979, 1980). This phase is often called Type I migration (Ward 1997ab, Tanaka et al. 2002) and can cause a forming planet to be accreted onto its central star in about 0.1 - $1 \mathrm{Myr}$, time scales shorter than the expected time $(1-$ $10 \mathrm{Myr}$ ) required for Jovian planets to attain their final masses (e.g., Lissauer \& Stevenson 2007). However, if a growing planet can attain a mass greater than $\sim 30-100 M_{\oplus}$ before accretion, it can clear a gap in the disk, and its subsequent migration rate is much smaller (this latter process is known as Type II migration). We note that the mass required for gap clearing depends on viscosity, scale height, and other disk parameters, so that a range of values is expected (for further detail, see Ward 1997a, especially Figure 14). In any case, the forming planet must grow massive enough - quickly enough - in order to survive. The problem is made more urgent because the Type I migrate rate increases with increasing planetary mass until the gap-clearing threshold is reached. This dilemma is generally known as the "Type I Migration Problem" and can be alleviated by the action of stochastic torques produced by disk turbulence. These torques drive random walk behavior that allows some fraction of the growing planetary cores to survive. The goal of this paper is to study Type I migration in the presence of stochastic torques in order to assess the expected survival rates for forming planets and to elucidate the physics of this mechanism.

A significant body of previous work exists. Initial explorations of the effects of turbulence on Type I migration showed that stochastic torques can dominate the steady inward torques and thus have the potential to allow more planets to survive (Laughlin et al. 2004, hereafter LSA; Nelson \& Papaloizou 2004, hereafter NP). Subsequent numerical studies demonstrated the corresponding random walk behavior of the migrating planets and explored the possible range of turbulent fluctuation amplitudes and correlation times (Nelson 2005, Papaloizou et al. 2007). Due to computational limitations, however, full numerical simulations that simultaneously include MHD turbulence and planetary migration can only be carried out for hundreds of orbits, whereas the expected time scale of interest is millions of years (and hence millions of orbits). As a result, long term behavior must be studied using analytical and statistical methods. Preliminary results were given in LSA, and then a more comprehensive treatment using Fokker-Planck methods was developed (Johnson et al. 2006, hereafter JGM). This latter work showed that only a small fraction of the planet population is expected to survive in the long term, and also considered the effects of disk structure on the results (e.g., departures from power-law surface density and temperature profiles — see also Menou \& Goodman 2004). This present paper also adopts an analytical/statistical approach in order to study the long-term outcome of this migration mechanism. Our goal is thus to generalize the previous analyses of LSA and JGM. 
This paper extends previous work in several ways: We explore the effects of the outer boundary condition. In particular, if the disk has an outer edge, as expected for young star/disk systems (typically with $r_{\text {disk }} \sim 30-100 \mathrm{AU}$ ), the outer boundary condition affects the dynamics by enforcing exponential decay in the number of surviving planets. In contrast, the survival fraction decays as a power-law decay in the limit where $r_{\text {disk }} \rightarrow \infty$. We also consider the effects of the initial conditions on the survival rates; planets formed in the outer disk have a much greater chance of survival, compared with those formed in the inner disk, with the boundary close to $r \sim 10$ AU (near the expected locations for planetary cores to form). Next we consider the possible effects of time dependence on the migration torques. Over the time span of interest, millions of years, the disk mass and the disk surface density decrease with time, whereas the mass of the migrating planetary core will grow. Both of these effects lead to time varying torque parameters, which are modeled herein. In the long time limit, we find the distribution of surviving planets by solving for the lowestorder eigenfunction of the Fokker-Planck equation. The dynamics of this migration problem are surprisingly rich. For example, although turbulent torques lead to random walk behavior and allow planets to survive, large amplitude fluctuations actually reduce the survival fraction; we explore the interplay between these competing outcomes and solve the corresponding optimization problem. Finally, we present an iterative map approach. In addition to providing an alternate description for the dynamics of the migration problem, this approach easily allows for the inclusion of eccentricity variations and large fluctuations.

This paper is organized as follows. We present our formulation of the Type I migration torques and turbulent forcing in Section 2. Section 3 develops a Fokker-Planck approach to the dynamics, including the basic formulation, analytic results for the cases where inward migration and diffusion are considered in isolation, as well as a self-similar model. Numerical solutions to the Fokker-Planck equation are presented in Section 4, which contains the main astronomical results (outlined above). The paper concludes in Section 5 with a summary of our results and a discussion of their implications. The Appendix presents an alternate approach to the migration problem using an iterative mapping scheme; this treatment not only adds to our understanding of the underlying dynamics, it can also be used to include larger stochastic perturbations, different boundary conditions, and additional variables.

\section{FORMULATION}

\subsection{Basic Disk Properties}

In order to explore the wide range of possible effects that arise in this coupled migration problem, we consider simple power-law disk models. Specifically, the surface density and temperature distribution of the disks are taken to be power-laws in radius,

$$
\Sigma(r)=\Sigma_{1}\left(\frac{r_{1}}{r}\right)^{p} \quad \text { and } \quad T(r)=T_{1}\left(\frac{r_{1}}{r}\right)^{q} .
$$


The normalization constants are determined by the total disk mass and total effective disk luminosity, respectively. In this formulation, we take $r_{1}=1 \mathrm{AU}$, so the coefficients $\Sigma_{1}$ and $T_{1}$ correspond to their values at $1 \mathrm{AU}$. The index $p$ is expected to lie in the range $p=1-2$, with a typical value $p=3 / 2$. This latter value arises from the Minimum Mass Solar Nebula (e.g., Weidenschilling 1977). Considerations of disk formation during protostellar collapse produce indices in the range $p=3 / 2-7 / 4$ (Cassen \& Moosman 1981, Adams \& Shu 1986). The normalization for the surface density has a benchmark value of $\Sigma_{1} \approx 4500 \mathrm{~g} / \mathrm{cm}^{2}$ (e.g., Kuchner 2004, Weidenschilling 1977). The power law index of the temperature profile is expected to be $q \approx 3 / 4$ for a viscous accretion disk (e.g. Pringle 1981) and a flat reprocessing disk (Adams \& Shu 1986), whereas $q \approx 1 / 2$ for a flared reprocessing disk (Chiang \& Goldreich 1997). The latter value also applies to the early solar nebula (Weidenschilling 1977).

The disk is assumed to be purely Keplerian, and the orbits are taken to be circular, so that the orbital angular momentum $j$ is given by

$$
j=m_{P}\left(G M_{*} r\right)^{1 / 2} .
$$

Further, the disk scale height $H$ is given by $H=a_{S} / \Omega$, where $a_{S}$ is the sound speed, which is in turn determined by the disk temperature profile. As shown below, the formulation of this paper requires specification of the scale height, rather than the temperature distribution itself, and we adopt the form

$$
\frac{H}{r}=\left(\frac{H}{r}\right)_{1}\left(\frac{r}{r_{1}}\right)^{(1-q) / 2} .
$$

A benchmark value for the scale height at $r_{1}=1 \mathrm{AU}$ is $H / r=0.1$.

\subsection{Turbulent Forcing}

The net effect of turbulence is to provide stochastic forcing perturbations. We first specify the time scale $\tau_{T}$ required for the disk to produce an independent realization of the turbulent fluctuations. Previous work (LSA, NP, Nelson 2005) indicates that this time scale is approximately an orbit time, so we parameterize the time scale according to

$$
\tau_{T}=f_{\alpha} \frac{2 \pi}{\Omega}
$$

where $\Omega$ is the Keplerian rotation rate and where $f_{\alpha}$ is a dimensionless parameter of order unity. Note that this time scale varies with radial location in the disk.

Next we need to determine the amplitudes $[(\Delta j) / j]_{k}$ of the angular momentum perturbations due to turbulent forcing. In general, the torque exerted on a planet by the disk will be a fraction of the benchmark scale $T_{D}$ given by

$$
T_{D}=2 \pi G \Sigma r m_{P}
$$


where $\Sigma$ is the disk surface density (e.g., JGM). The amplitude for angular momentum variations is thus given by

$$
\Delta j=f_{T} T_{D} \tau_{T}
$$

where $\tau_{T}$ is the time over which one independent realization of the turbulence acts. The total torque produced by the turbulence is a fraction $f_{T}$ of the benchmark scale given by equation (5). These turbulent forcing amplitudes have been estimated using MHD simulations (e.g., LSA, NP, Nelson 2005), which show that $f_{T} \sim 0.05$ (with a range of variation about this typical value). The relative fluctuation amplitude is then given by

$$
\left(\frac{\Delta j}{j}\right)_{T}=f_{\alpha} f_{T}(2 \pi)^{2} \frac{\Sigma r^{2}}{M_{*}}
$$

With $f_{T}=0.05$ and $f_{\alpha}=1$, the leading numerical coefficient becomes $\pi^{2} / 5 \sim 2$. The expression in equation (7) determines the fluctuation amplitude. The actual changes in angular momentum over a given time scale $\tau_{T}$ are thus given by

$$
\frac{\Delta j}{j}=\left(\frac{\Delta j}{j}\right)_{T} \xi=f_{\alpha} f_{T}(2 \pi)^{2} \frac{\Sigma r^{2}}{M_{*}} \xi,
$$

where the random variable $\xi$ has zero mean and unit variance. In this work, we assume that $\xi$ has a gaussian distribution.

Note that this treatment also assumes that the planet is small enough so that it has no back reaction on the disk. Since we are primarily interested in planetary cores in the mass range $m_{P}=$ $1-30 M_{\oplus}$, this assumption is expected to be valid. Planets of larger mass are likely to clear gaps in their immediate vicinity within the disk (Goldreich \& Tremaine 1980), however, and hence the turbulent torques are reduced in such systems (this reduction can be included in the formalism; see Adams et al. 2008).

For power-law disks, the relative fluctuation amplitude varies with radius according to

$$
\left(\frac{\Delta j}{j}\right)_{T} \propto r^{2-p}
$$

For a typical value of the power-law index is $p=3 / 2$, the relative fluctuations $[(\Delta j) / j] \sim r^{1 / 2} \sim j$.

\subsection{Type I Migration}

The strength of Type I torques are given by

$$
T_{1}=f_{1}\left(\frac{m_{P}}{M_{*}}\right)^{2} \pi \Sigma r^{2}(r \Omega)^{2}\left(\frac{r}{H}\right)^{2}
$$

where $f_{1}$ is a dimensionless (constant) parameter (Ward 1997a). Over the same time scale $\tau_{T}$ used to evaluate the changes in angular momentum due to turbulence, the corresponding changes due 
to Type I torques are given by

$$
\left(\frac{\Delta j}{j}\right)_{1}=f_{1} f_{\alpha} 2 \pi^{2}\left(\frac{m_{P}}{M_{*}}\right) \frac{\Sigma r^{2}}{M_{*}}\left(\frac{r}{H}\right)^{2} .
$$

For power-law disks, the Type I angular momentum increments vary with radius according to

$$
\left(\frac{\Delta j}{j}\right)_{1} \propto r^{1+q-p}
$$

For typical indices $p=3 / 2$ and $q=3 / 4$, the Type I angular momentum increments vary relatively slowly with radius, i.e., $[(\Delta j) / j] \sim r^{1 / 4} \sim j^{1 / 2}$. For the particular values $p=3 / 2$ and $q=1 / 2$, often used to model the early solar nebula, the relative fluctuation $[(\Delta j) / j]$ is a constant with respect to radius $r$. For typical values of the input parameters, the constant amplitude of the angular momentum increment is given by $[(\Delta j) / j] \sim 10^{-5}$.

\subsection{Comparison of Time Scales}

The Type I migration torques provide a steady inward forcing on the planets, whereas the turbulent torques are stochastic. At a given radial location in the disk, or equivalently at a given value of angular momentum $j$, the ratio of the time scales for the two types of torques to move the planet is given by

$$
\frac{t_{1}}{t_{T}}=\frac{[(\Delta j) / j]_{T}^{2}}{[(\Delta j) / j]_{1}}=\frac{8 \pi^{2} f_{\alpha} f_{T}^{2}}{f_{1}}\left(\frac{\Sigma H^{2}}{m_{P}}\right) .
$$

The expected value of the leading coefficient is $\sim 1 / 5$. For power-law disks, this ratio of time scales varies with radius according to

$$
\frac{t_{1}}{t_{T}} \propto r^{3-p-q} .
$$

As a result, the time scale ratio grows (approximately) linearly with radius. More significantly, the power-law index is always positive, even for the most extreme parameters expected in planetforming disks, so that the outer disk is dominated by turbulent migration, while the inner disk is dominated by Type I migration.

The above discussion motivates the definition of a dimensionless parameter $Q_{\mathrm{m}}$ that determines the characteristics of planetary migration at a given radial location in the disk:

$$
Q_{\mathrm{m}} \equiv \frac{8 \pi^{2} f_{T}^{2} \Sigma H^{2}}{m_{P}}
$$

where we have ignored the parameters $f_{1}$ and $f_{\alpha}$ since they are expected to be close to unity. For $Q_{\mathrm{m}}>1$, turbulent torques dominate and migration behaves as a random walk. For $Q_{\mathrm{m}}<1$, Type I torques dominate and planets migrate steadily inward. For typical disk parameters, we expect $Q_{\mathrm{m}} \sim 0.1$ near $r=1 \mathrm{AU}$. Keep in mind that the value of $Q_{\mathrm{m}}$ depends on both the radial location 
in the disk and on time. As the disk and planet evolve, the surface density $\Sigma$ grows smaller, while the planetary core mass $m_{P}$ grows larger, so that $Q_{\mathrm{m}}$ is generally a decreasing function of time.

On a related note, we can estimate the time required for the two migration mechanisms to move planets from a given starting point in the disk to either the inner or outer disk edge. The time required for Type I migration to move a planet inward to the star from a starting angular momentum value $j_{0}$ is approximately given by $t_{I} \approx j_{0} /\left[3 T_{1}\left(j_{0}\right)\right]$. To fix ideas, we take the starting radius to be $10 \mathrm{AU}$. For typical values of the torque parameters, the Type I time scale for inward migration is $t_{I} \sim 1 \mathrm{Myr}$. For comparison, we can estimate the time required for diffusion to transport planets to the outer disk edge. The timescale for the distribution to spread to the outer edge is given by $t_{T} \approx N \tau_{T} \approx\left\langle\tau_{T}\right\rangle\left[j_{\text {disk }} /(\Delta j)_{T}\right]^{2}$, where $j_{\text {disk }}$ is the angular momentum at the outer disk edge $r_{\text {disk }}$. If we take $r_{\text {disk }}=100 \mathrm{AU}$, with corresponding angular momentum $j_{\text {disk }}$, the timescale $t_{T} \sim 8 \mathrm{Myr}$. However, the time required for the first planet to reach the outer edge can be much shorter $(\sim 0.1 \mathrm{Myr})$. Edge effects start to be important at an intermediate time scale, i.e., about $1 \mathrm{Myr}$ (from the geometric mean).

These time scales thus frame the problem: Since the Type I migration time scale is roughly comparable to - but shorter than - the outward diffusion time scale, the population of planetary cores is expected to be highly depleted, even though diffusion acts to save some fraction of them. In addition, the diffusion time scale is comparable to expected disk lifetimes, so that the outer disk edge will have an important impact on the results. Keep in mind that the time scales quoted here depend on the starting radius, the outer disk radius, and the size of the torque parameters, so that a range of values will be applicable to the actual population of planet-forming disks. In particular, if the starting radius is larger (than $10 \mathrm{AU}$ as assumed above), the outward diffusion time scale will decrease and the inward Type I migration time will increase.

\section{FOKKER-PLANCK TREATMENT: ANALYTIC RESULTS}

After formulating this planet migration problem in terms of a Fokker-Planck equation (Section 3.1), we explore analytic solutions. If we consider either Type I migration torques (Section 3.2) or the turbulent diffusion (Section 3.3) acting alone, the resulting dynamics can be solved exactly. We also construct a self-similar model of the diffusion process (Section 3.4) that applies in the absence of an outer edge to the disk. These cases - exact solutions to partial versions of the problem provide us with an understanding of the relevant physical mechanisms. On the other hand, they do not provide reliable estimates for the planetary survival probabilities; these quantities are thus determined numerically in Section 4. 


\subsection{Formulation}

Let $P(j, t)$ denote the distribution of an ensemble of planets as a function of time. The general form of the Fokker-Planck equation (e.g., Risken 1984) for this problem is given by

$$
\frac{\partial P}{\partial t}-\frac{\partial}{\partial j}\left[T_{1}(j) P\right]=\frac{\partial^{2}}{\partial j^{2}}[D(j) P]
$$

where $T_{1}(j)$ is the Type I migration torque and $D(j)$ is the appropriate diffusion parameter due to turbulent fluctuations. In this problem (see also JGM), the diffusion constant is defined to be $D \equiv(\Delta J)_{T}^{2} / \tau_{T}$, where the fluctuation amplitude $(\Delta J)_{T}$ and the time scale $\tau_{T}$ over which the turbulent perturbations are independent are specified in Section 2.2 (see equations 4- 7). Notice also that the minus sign in the Type I term is included so that $T_{1}$ is the magnitude of the torque.

Next we want to formulate the problem in terms of simplified quantities. We define a dimensionless angular momentum variable

$$
x \equiv j / j_{1},
$$

where $j_{1}$ is the angular momentum at a convenient reference location; for the sake of definiteness we take $j_{1}=j\left(r_{1}\right)$, where $r_{1}=1 \mathrm{AU}$. For most cases of interest, both the torque $T_{1}(j)$ and the diffusion "constant" $D(j)$ are functions of angular momentum. If we specialize to the case where the disk surface density and temperature profile are power-laws in radius (equation [1]), we can write $T_{1} \propto j^{-a}$ and $D \propto j^{b}$, where $a=2$ and $b=1$ for standard disk parameters. In general, the indices are given by

$$
a=2(p-q) \quad \text { and } \quad b=7-4 p,
$$

where $p$ and $q$ are the power-law indices of the disk surface density and temperature profiles, respectively. Next we define a reduced Type I torque constant $\gamma$ and a reduced diffusion constant $\beta$,

$$
\gamma \equiv \frac{T_{1}\left(j_{1}\right)}{j_{1}} \quad \text { and } \quad \beta \equiv \frac{D\left(j_{1}\right)}{j_{1}^{2}}
$$

The general form of the Fokker-Planck equation thus becomes

$$
\frac{\partial P}{\partial t}=\gamma \frac{\partial}{\partial x}\left[x^{-a} P\right]+\beta \frac{\partial^{2}}{\partial x^{2}}\left[x^{b} P\right] .
$$

Note that both of the constants $\gamma$ and $\beta$ are rates, i.e., they have units of (time) $)^{-1}$. The Type I migration parameter $\gamma$ takes the form

$$
\gamma=\left[\pi f_{1}\left(\frac{m_{P}}{M_{*}}\right)\left(\frac{r}{H}\right)^{2} \frac{G \Sigma r}{\sqrt{G M_{*} r}}\right]_{1 \mathrm{AU}},
$$

where the subscript specifies that all quantities are evaluated at $r=1 \mathrm{AU}$. For typical values of the surface density at $1 \mathrm{AU}, \Sigma_{1}=4500 \mathrm{~g} / \mathrm{cm}^{2}$, the scale height $(H / r)_{1}=0.1$, and for $m_{P}=\sqrt{10} M_{\oplus}$, the parameter $\gamma \approx 10^{-5} \mathrm{yr}^{-1}=10 \mathrm{Myr}^{-1}$. The reduced diffusion parameter $\beta$ has the form

$$
\beta=\left[f_{\alpha} f_{T}^{2}(2 \pi)^{3}\left(\frac{\Sigma r^{2}}{M_{*}}\right)^{2} \Omega\right]_{1 \mathrm{AU}} .
$$


For the same disk parameters quoted above, the value of the diffusion parameter $\beta \approx 10^{-6} \mathrm{yr}^{-1}$ $=1 \mathrm{Myr}^{-1}$. The corresponding time scales are thus given by $1 / \gamma \sim 0.1 \mathrm{Myr}$ and $1 / \beta \sim 1 \mathrm{Myr}$. Notice that the ratio of the Type I torque parameter to the diffusion parameter is the ratio of time scales given by equation (13) so that $\beta / \gamma=Q_{\mathrm{m}}$ (see equation [15).

In this treatment, the Fokker-Planck equation (20) does not contain a source term. Although a given circumstellar disk may produce multiple planetary cores, it will not produce a statistically significant distribution of cores. The distribution function $P(t, x)$ thus represents the output from a large ensemble of planet-forming disks, all with the same properties. Since this treatment does not include planet-planet interactions, multiple cores in a particular disk will act (statistically) as part of this same ensemble.

In addition to specifying the disk properties, we must also specify the initial conditions, which is determined by the initial distribution of planets $P(t=0, x)$. For most of this work, we take the initial distribution to be a narrow gaussian centered on a given value of angular momentum $x_{0}$. Realistic disks will produce planetary cores at a range of radial locations and hence a range of $x_{0}$. By taking the initial conditions to be a narrow gaussian, we are thus studying the effects of one starting point at a time.

Finally, we must specify the boundary conditions. At the outer edge of the disk, corresponding to the maximum value $x_{\max }$ of dimensionless angular momentum, we assume that the probability current $S(x, t)$ must vanish. This condition is equivalent to that of requiring "zero flux" through the outer boundary and can be written in the form

$$
S\left(x_{\max }, t\right)=-\left[\gamma x^{-a} P+\beta \frac{\partial}{\partial x}\left(x^{b} P\right)\right]_{x_{\max }}=0,
$$

where the current $S(x, t)$ is determined by the right hand side of the Fokker-Planck equation (201). In physical terms, this boundary condition assumes that no planets can migrate beyond the regions where disk material resides, and that no planets enter the disk from large radii (see JGM for further discussion of this issue). At the inner boundary, we use the ansatz $P=$ constant, the form appropriate for an "absorbing wall" (Risken 1984). This inner boundary condition thus assumes that planetary cores are accreted once they reach the star.

The survival probability for planets, and other results of interest, depend on this choice for the outer boundary condition, as well as the location of the outer boundary. For the special case of self-similar solutions (Sections 3.4 and 4.2), we take the limit $x_{\max } \rightarrow \infty$ and apply the boundary condition (23) there. For disks with a finite radial extent, our boundary condition at the outer disk edge represents a "reflecting wall" (Risken 1984). Note that it remains possible for planetary cores near the outer edge to be scattered outside the disk by turbulence. Once outside the disk material, these planets would become stranded. If the disk edge moves out with time, due to viscous spreading, stranded planets could be pushed further outwards. Although planetary cores could be "saved" in this manner, they would be unlikely to form giant planets because of the lack of gas and the long orbit times (both of which inhibit giant planet formation). In addition, the relative 
amplitude is small at the outer edge, $([\Delta J] / J)_{T} \approx 10^{-2}$ (see equation [7), so that such events could be rare. However, if Type I migration can reverse its direction and move planets outward (as suggested by Paardekooper \& Mellema 2006), then this mechanism could be important. To include this effect in the calculations, one would use an absorbing boundary condition at the outer edge (or a partial barrier); this choice would allow more planetary cores to survive, but would result in the formation of fewer giant planets. For the relatively short timescales of interest here ( $\sim 10 \mathrm{Myr}$ ), the choice of boundary condition produces modest differences; however, the predicted survival probabilities would be affected over sufficiently long times.

As written, equation (20) contains two parameters $(\gamma, \beta)$ that set the strength of the torques and two indices $(a, b)$ that determine their radial dependence. Although this formulation thus results in a four-dimensional parameter space, its size can be reduced. First, we note that the indices $(a, b)$ have relatively limited ranges, and that the effects of turbulence always grow with radius compared to Type I torques. As a result, we fix the indices to their "standard" values $(a, b)=(1,2)$ for much of our exploration. For given values of the indices, one of the remaining variables can be scaled out of the problem by changing the definition of time. For example, let $t \rightarrow \gamma t$, and $\beta \rightarrow \beta / \gamma=Q_{\mathrm{m}}$. In this case, time is measured in units of the Type I migration time (typically several Myr) and $Q_{\mathrm{m}}=\beta / \gamma$ defines the level of turbulence relative to the Type I torque strength (at $1 \mathrm{AU}$ ). In this reduced view, the Fokker-Planck equation has a one parameter family of solutions, and that parameter can be taken to be $Q_{\mathrm{m}}$ as defined by equation (15). In the limit $Q_{\mathrm{m}} \rightarrow 0$, Type I torques dominate the migration process, and fully analytic solutions can be obtained (see Section 3.2). In the opposite limit $Q_{\mathrm{m}} \rightarrow \infty$, turbulent torques dominate, and analytic solutions can once again be constructed (Section 3.3).

\subsection{Solutions with Only Inward Migration}

This section considers the limit $Q_{\mathrm{m}} \rightarrow 0$ where Type I torques dominate. In terms of the reduced quantities defined above, the Fokker-Planck equation in the absence of diffusion has the form

$$
\frac{\partial P}{\partial t}=\gamma \frac{\partial}{\partial x}\left[\frac{P}{x^{a}}\right]
$$

General solutions of this equation can be found by making the following transformation of both the angular momentum variable $x$ and the function $P$ itself:

$$
z \equiv \frac{x^{a+1}}{a+1} \quad \text { and } \quad f(z, t)=x^{-a} P[x(z), t] .
$$

With this change of variables, the equation of motion becomes

$$
\frac{\partial f}{\partial t}=\gamma \frac{\partial f}{\partial z}
$$

which has solutions of the form

$$
f=f(z+\gamma t) \quad \text { and } \quad P=x^{a} f(z+\gamma t),
$$


where $z$ is related to $x$ through equation (25). The form of the function $f$ is specified by the initial condition, so that

$$
f(z)=x^{-a} P(x, 0)
$$

To illustrate this type of solution, we consider the case where the initial distribution of angular momentum has a gaussian form, i.e.,

$$
P(x, 0)=\frac{1}{\sigma \sqrt{\pi}} \exp \left[-\frac{\left(x-x_{0}\right)^{2}}{\sigma^{2}}\right],
$$

where $x_{0}$ is the angular momentum at the peak of the initial distribution. Note that the distribution is normalized over positive angular momentum values $x$ and has width given by $\sigma$. The timedependent solution thus has the form

$$
P(x, t)=\frac{1}{\sigma \sqrt{\pi}} \frac{x^{a}}{\left[x^{a+1}+(a+1) \gamma t\right]^{a /(a+1)}} \exp \left[-\frac{\left\{\left[x^{a+1}+(a+1) \gamma t\right]^{1 /(a+1)}-x_{0}\right\}^{2}}{\sigma^{2}}\right] .
$$

The probability $p_{S}(t)$ of planet survival can be found by integrating the solution given by equation (30) over all positive values of $x$. In the limit where the width of the initial distribution is small compared to the peak, and the time of observation is long, the parameters of the problem obey the ordering

$$
[(a+1) \gamma t)]^{1 /(a+1)} \gg x_{0} \gg \sigma .
$$

In this limit, the survival probability can be written in the form

$$
p_{S}(t)=\frac{1}{2} \operatorname{Erfc}\left[\frac{[(a+1) \gamma t)]^{1 /(a+1)}}{\sigma}\right] \approx \frac{\sigma}{2 \sqrt{\pi}[(a+1) \gamma t)]^{1 /(a+1)}} \exp \left[-\frac{[(a+1) \gamma t)]^{2 /(a+1)}}{\sigma^{2}}\right],
$$

where $\operatorname{Erfc}(x)$ is the complementary error function (AS), and where the second approximate equality holds in the asymptotic limit.

\subsection{Solutions with Only Diffusion}

This section considers the opposite limit where $Q_{\mathrm{m}} \rightarrow \infty$, i.e., we neglect the Type I migration torques so that $\gamma=0$. We make the additional restriction to the case where the diffusion constant $D(j) \propto j$ (so that $b=1$ ); as a result, this treatment is not as general as that of Section 3.2. If we redefine the time variable so that $t \rightarrow \beta t$, the diffusion equation for the probability distribution takes the form

$$
\frac{\partial P}{\partial t}=\frac{\partial^{2}}{\partial x^{2}}[x P]
$$

Note that $t$ is a dimensionless time variable, or, equivalently, time is measured in units of the diffusion timescale. 
If we separate the diffusion equation so that $P(x, t)=G(t) F(x)$, the temporal solutions take the form

$$
G(t)=\exp [-\lambda t]
$$

where $\lambda$ is the separation constant, and the remaining differential equation for $F(x)$ becomes

$$
x \frac{d^{2} F}{d x^{2}}+2 \frac{d F}{d x}+\lambda F=0 .
$$

After some rearrangement, the solution to equation (35) can be written in the form

$$
F(x)=\frac{1}{\sqrt{\lambda x}} J_{1}(2 \sqrt{\lambda x})
$$

where $J_{1}(x)$ is the Bessel Function of the first kind of order one (Abramowitz \& Stegun 1970; hereafter AS). This solution is chosen to be finite at the origin $x=0$. To apply the outer boundary condition, we require that the flux at the outer edge of the disk vanish. This location corresponds to a maximum value $x_{\max }$ of the dimensionless angular momentum. After defining $\xi \equiv 2 \sqrt{\lambda x}$, the outer boundary condition (see equation [23]) takes the form

$$
\frac{d}{d x}[x F(x)]=0 \quad \Rightarrow \quad \frac{d}{d \xi}\left[\xi J_{1}(\xi)\right]=0=\xi J_{0}(\xi),
$$

where we have used the properties of Bessel functions (AS) to obtain the final equality. The separation constant must be chosen so that the outer boundary occurs at a zero of the zeroth order Bessel function $J_{0}$. If we denote the zeroes of $J_{0}$ by $\xi_{\nu}$, the separation constants $\lambda_{\nu}$ are given by

$$
\lambda_{\nu}=\xi_{\nu}^{2} / 4 x_{\max }
$$

The general solution thus takes the form

$$
P(x, t)=\sum_{\nu=1}^{\infty} A_{\nu} \exp \left[-\lambda_{\nu} t\right] \frac{1}{\sqrt{\lambda_{\nu} x}} J_{1}\left(2 \sqrt{\lambda_{\nu} x}\right)
$$

where the $\lambda_{\nu}$ are given by equation (38). Suppose we are given an initial distribution $f(x)$ at $t=$ 0, i.e.,

$$
P(x, t=0)=\sum_{\nu=1}^{\infty} A_{\nu} \frac{1}{\sqrt{\lambda_{\nu} x}} J_{1}\left(2 \sqrt{\lambda_{\nu} x}\right)=f(x) .
$$

Next we multiply both sides of the equation by $\sqrt{x} J_{1}\left(2 \sqrt{\lambda_{\mu} x}\right)$ and then integrate:

$$
\sum_{\nu=1}^{\infty} \frac{A_{\nu}}{\sqrt{\lambda_{\nu}}} \int_{0}^{x_{\max }} d x J_{1}\left(2 \sqrt{\lambda_{\nu} x}\right) J_{1}\left(2 \sqrt{\lambda_{\mu} x}\right)=\sum_{\nu=1}^{\infty} \frac{A_{\nu}}{\sqrt{\lambda_{\nu}}} I_{\nu}=\int_{0}^{x_{\max }} d x \sqrt{x} f(x) J_{1}\left(2 \sqrt{\lambda_{\mu} x}\right) .
$$

The integrals $I_{\nu}$ in the sum can then be rewritten by changing variables to $u^{2}=x / x_{\max }$, so they take the form

$$
I_{\nu}=2 x_{\max } \int_{0}^{1} u d u J_{1}\left(\xi_{\nu} u\right) J_{1}\left(\xi_{\mu} u\right)
$$


where the $\xi_{k}$ are zeroes of the $J_{0}$ functions. After applying the recursion relations for Bessel functions and integrating by parts, we find

$$
I_{\nu}=2 x_{\max } \frac{\xi_{\nu}}{\xi_{\mu}} \int_{0}^{1} u d u J_{0}\left(\xi_{\nu} u\right) J_{0}\left(\xi_{\mu} u\right)=x_{\max }\left[J_{1}\left(\xi_{\nu}\right)\right]^{2} \delta_{\nu \mu}
$$

The coefficients $A_{\nu}$ can now be evaluated:

$$
A_{\nu}=\frac{\sqrt{\lambda_{\nu}}}{\left[J_{1}\left(\xi_{\nu}\right)\right]^{2}} \frac{1}{x_{\max }} \int_{0}^{x_{\max }} d x \sqrt{x} f(x) J_{1}\left(2 \sqrt{\lambda_{\nu} x}\right)
$$

As one example, we consider the case in which all of the planets start at the same radius, or angular momentum, so that the starting distribution $f(x)=\delta\left(x-x_{0}\right)$, and the $A_{\nu}$ take the form

$$
A_{\nu}=\frac{\sqrt{\lambda_{\nu} x_{0}}}{x_{\max }\left[J_{1}\left(\xi_{\nu}\right)\right]^{2}} J_{1}\left(2 \sqrt{\lambda_{\nu} x_{0}}\right)=\frac{\xi_{\nu} \sqrt{x_{0} / x_{\max }}}{2 x_{\max }\left[J_{1}\left(\xi_{\nu}\right)\right]^{2}} J_{1}\left(\xi_{\nu} \sqrt{x_{0} / x_{\max }}\right) .
$$

Next, we can find the total survival probability by starting with the full time dependent solution of equation (39) and integrating over all angular momentum values,

$p_{S}(t)=\int_{0}^{x_{\max }} P(x, t) d x=\sum_{\nu=1}^{\infty} A_{\nu} \exp \left[-\lambda_{\nu} t\right] \int_{0}^{x_{\max }} \frac{d x}{\sqrt{\lambda_{\nu} x}} J_{1}\left(2 \sqrt{\lambda_{\nu} x}\right)=\sum_{\nu=1}^{\infty} \frac{4 x_{\max } A_{\nu}}{\xi_{\nu}^{2}} \exp \left[-\lambda_{\nu} t\right]$.

For example, for the particular case in which the starting angular momentum distribution is a delta function, so that the coefficients $A_{\nu}$ are given by equation (45), the probability takes the form

$$
p_{S}(t)=\sum_{\nu=1}^{\infty} \frac{2 u_{0} J_{1}\left(\xi_{\nu} u_{0}\right)}{\xi_{\nu}\left[J_{1}\left(\xi_{\nu}\right)\right]^{2}} \exp \left[-\lambda_{\nu} t\right]
$$

where $u_{0} \equiv\left(x_{0} / x_{\max }\right)^{1 / 2}$.

In this problem, the flux at the outer boundary vanishes, and the flux into the origin is given by

$$
\begin{aligned}
\mathcal{F}_{0}= & -\left.\frac{\partial}{\partial x}(x P)\right|_{x=0}=-\sum_{\nu=1}^{\infty} A_{\nu} \exp \left[-\lambda_{\nu} t\right] \frac{1}{\sqrt{\lambda_{\nu}}} \frac{\partial}{\partial x}\left[x^{1 / 2} J_{1}\left(2 \sqrt{\lambda_{\nu} x}\right)\right]_{x=0} \\
= & -\sum_{\nu=1}^{\infty} A_{\nu} \exp \left[-\lambda_{\nu} t\right]\left[\frac{J_{1}\left(x_{\nu}\right)}{x_{\nu}}+\frac{d J_{1}}{d x}\right]_{x_{\nu}=0}=-\sum_{\nu=1}^{\infty} A_{\nu} \exp \left[-\lambda_{\nu} t\right]
\end{aligned}
$$

where $x_{\nu}=2 \sqrt{\lambda_{\nu} x}$. For comparison,

$$
\frac{d p_{S}}{d t}=-\sum_{\nu=1}^{\infty} A_{\nu} \frac{4 x_{\max } \lambda_{\nu}}{\xi_{\nu}^{2}} \exp \left[-\lambda_{\nu} t\right]=-\sum_{\nu=1}^{\infty} A_{\nu} \exp \left[-\lambda_{\nu} t\right]
$$

Thus, $d p_{S} / d t=\mathcal{F}_{0}$, as expected. 
At late times, only the leading term survives in the series that describes the solutions. As a result, the first term of equation (39) determines the probability distribution in the long time limit. As a result, the distribution of locations for surviving planetary cores is given by the first order Bessel function of the first kind. Similarly, the total survival probability is given by the first term in equation (47). The first three zeroes (of the zeroth Bessel function of the first kind) are $\xi_{1} \approx$ $2.40482, \xi_{2} \approx 5.52007$, and $\xi_{3} \approx 8.65372(\mathrm{AS})$; if we take the outer boundary to be $x_{\max }=10$ (corresponding to an outer disk radius of $100 \mathrm{AU}$ ), the first three eigenvalues (see equation [38]) are approximately $\lambda_{1} \approx 0.145, \lambda_{2} \approx 0.762$, and $\lambda_{3} \approx 1.87$. After one diffusion time scale (roughly $1 \mathrm{Myr}$ ), the first term is about twice as large as the second. After 10 diffusion times (about 10 Myr), the first term is almost 500 times larger. In the (expected) case in which the planetary core population is severely depleted, the distributions are thus determined primarily by the leading order terms. We exploit this property of the solutions in Section 4.3, which determines the lowest order eigenfunctions and eigenvalues for the full problem, including Type I migration.

\subsection{Self-Similar Solutions}

In the absence of an outer disk edge, self-similar solutions to the Fokker-Planck equation exist (JGM). Although we expect the disk radius to be finite, with typical radii $r_{\text {disk }} \sim 30-100$ AU, we can use self-similar solutions as an analytic model of the dynamics to gain further insight into the problem. One should keep in mind, however, that these solutions overestimate the probability of planetary survival.

In the limit of long times, the surviving planets tend to reside in the outer disk where inward migration due to Type I torques is relatively unimportant compared with diffusion. As shown previously, self-similar solutions exist in this regime when the Type I torques vanish (JGM). However, we can include an inward torque term, and still retain self-similarity, provided that we use an averaged torque so that the Fokker-Planck equation takes the form

$$
\frac{\partial P}{\partial t}=\langle\gamma\rangle \frac{\partial P}{\partial x}+\beta \frac{\partial^{2}}{\partial x^{2}}(x P)
$$

where $\langle\gamma\rangle$ is now an appropriate average over the disk (to remove the additional $x$-dependence in the torque term). Since Type I torques remove planets from the inner disk on a short time scale (compared with the disk lifetime), the effective value $\langle\gamma\rangle$ should be representative of the outer disk; as a reference point, we expect $\gamma \approx \beta(0.1 \beta)$ at $r \approx 10 \mathrm{AU}$ (100 AU). Although this model equation is simpler than the full problem, it retains the crucial feature that the relative importance of diffusion (compared with Type I migration) increases outwards. In addition, an analytic solution can be found (see below) and the optimization calculation (see Section 4.2) can be done explicitly.

This version of the Fokker-Planck equation (50) has the solution

$$
P(x, t)=A(\beta t+\sigma)^{-(2+\langle\gamma\rangle / \beta)} \exp [-x /(\beta t+\sigma)]
$$


where $\sigma$ is a constant that is determined by the initial width of the distribution, and where $A$ is a normalization constant. For standard normalization, the expectation value of the initial state is given by $\left\langle x_{0}\right\rangle=\sigma$. For planets starting near $r=10 \mathrm{AU}$, we expect $\sigma \sim x_{0} \sim \sqrt{10} \sim 3$. As written, the solution extends to spatial infinity $(x \rightarrow \infty)$, where the distribution function obeys a zero-flux outer boundary condition (equation [23). Keep in mind that the solution given in equation (51) is the simplest member of a sequence of self-similar solutions.

In this model, the probability of a planet remaining in the disk is given by integrating the above solution over all values of $x$,

$$
p_{S}(t)=\int_{0}^{\infty} d x P(x, t)=\frac{A}{(\beta t+\sigma)^{1+\langle\gamma\rangle / \beta}} \approx(1+\beta t / \sigma)^{-(1+\langle\gamma\rangle / \beta)},
$$

where $A$ is a normalization constant, and we have normalized the solution in the final equality so that the total probability is unity at $t=0$. This result provides an exact solution to the simplified problem posed by equation (50), but is only an approximation to the original physical problem (with spatially varying Type I torques) because the true solution is expected to approach the selfsimilar form of equation (51) only at late times. As a result, the normalization (defined here at $t$ $=0)$ can be different.

In the limit $\langle\gamma\rangle \rightarrow 0$, the survival fraction approaches the form $p_{S} \propto 1 / t$ (see JGM). When Type I torques are included, the power-law steepens and hence fewer planets survive. Notice that this solution represents an upper bound on the true survival fraction for two reasons: The inclusion of the outer boundary (at the disk edge) enforces exponential decay in the long term (see Sections 3.3, 4.3, and Figures 2 and 3). In addition, the Type I torques are approximated here with no spatial dependence, and hence take on the value appropriate in the outer disk; including the spatial dependence will increase their efficacy and hasten the removal of planets from the inner disk.

Nonetheless, we can obtain a working estimate for the survival fraction. For typical values $\beta$ $=1 \mathrm{Myr}^{-1},\langle\gamma\rangle / \beta=0.3$, distribution width $\sigma=3=\left\langle x_{0}\right\rangle$, and time $t=10 \mathrm{Myr}$, we find $p_{S} \approx 0.15$. For this time scale, we thus find that planetary survival is only moderately rare, at the level of ten percent, roughly consistent with the numerical calculations of the previous section. This estimate is somewhat higher, however, primarily due to the absence of the outer boundary.

\section{FOKKER-PLANCK TREATMENT: NUMERICAL RESULTS}

We can directly solve the Fokker-Planck equation using standard numerical methods; here we use a fully implicit method (e.g., see Press et al. 1990). The boundary conditions play an important role in determining the fraction of surviving planets as a function of time. For the calculations of this section, we adopt a standard set of boundary conditions and initial conditions in order to determine how the planet survival fractions depend on time and on the torque parameters $\gamma$ and $\beta$. The inner boundary is fixed at $x=x_{*}=0.1(r=0.01 \mathrm{AU})$ and the outer boundary is fixed at $x=x_{\max }=10\left(r=r_{\text {disk }}=100 \mathrm{AU}\right)$. The distribution function $P$ is chosen to have a constant value 
at the inner boundary; note that a constant value of $P$ allows for nonzero flux through the inner boundary. At the outer boundary, we use the zero-flux condition, which in this formulation is given by equation (23). The initial condition is chosen to be a narrow gaussian distribution centered on

$x=x_{p}=\sqrt{r_{0}}$, corresponding to radius $r_{0}$. We use $r_{0}=10 \mathrm{AU}$ as a benchmark value, but explore varying values.

Through numerical experimentation, we find that the width of the initial gaussian has relatively little effect, provided that it is much narrower than the disk size. The location of the peak determines two important time scales for the evolution of the probability distribution: (1) the time required for the Type I torques to move planets from the peak location inward to the star, and (2) the time required for diffusion to spread the distribution to the outer disk edge, where the outer boundary affects the dynamics.

\subsection{Basic Numerical Results}

The evolution of the probability distribution is illustrated in Figure1, The torque parameters are chosen to be near the center of the range of expected values with $1 / \gamma=0.1$ Myr and $1 / \beta=1$ Myr. The figure shows the distribution $P(r, t)$ as a function of radius $r$. Note that the calculations are done in terms of dimensionless angular momentum $x$, so that the function $P$ represents the probability density in $x$, i.e., $P=d p / d x$. In the figures of this paper, however, we plot the function $P$ versus radius $r=x^{2}$ AU (because we have better intuition for the meaning of radial locations in these disks). As expected, the distribution spreads out with time, and its area decreases as planets are lost through accretion onto the central star. The peak of the distribution actually moves outwards with time, even though Type I migration acts to move planets inward. Here, at the relatively late times shown, any planets that diffuse into the inner regions of the disk are quickly swept into the star, and thus do not contribute to the distribution at small radii. Notice that the edge of the probability distribution reaches the outer boundary in only about $1 \mathrm{Myr}$, so the effect of the outer disk edge plays an important role in determining planet survivability on this time scale (and longer).

For a given distribution $P(x, t)$ at a specific time, the fraction of surviving planets $p_{S}(t)$ is given by the integral

$$
p_{S}(t)=\int_{x_{*}}^{x_{\max }} P(x, t) d x .
$$

Figure 2 shows the total probability of planet survival as a function of time for varying values of the Type I migration torques and fixed amplitude of the turbulent torques (with $\beta=1 \mathrm{Myr}^{-1}$ ). Figure 2 is presented as a log-linear plot, so that exponential decay corresponds to straight lines in the diagram. Note that all of the curves become straight lines asymptotically with time, so that the decay rate is in fact well-defined.

The solutions depicted in Figure 2 provide estimates for the survival probability. In the absence 


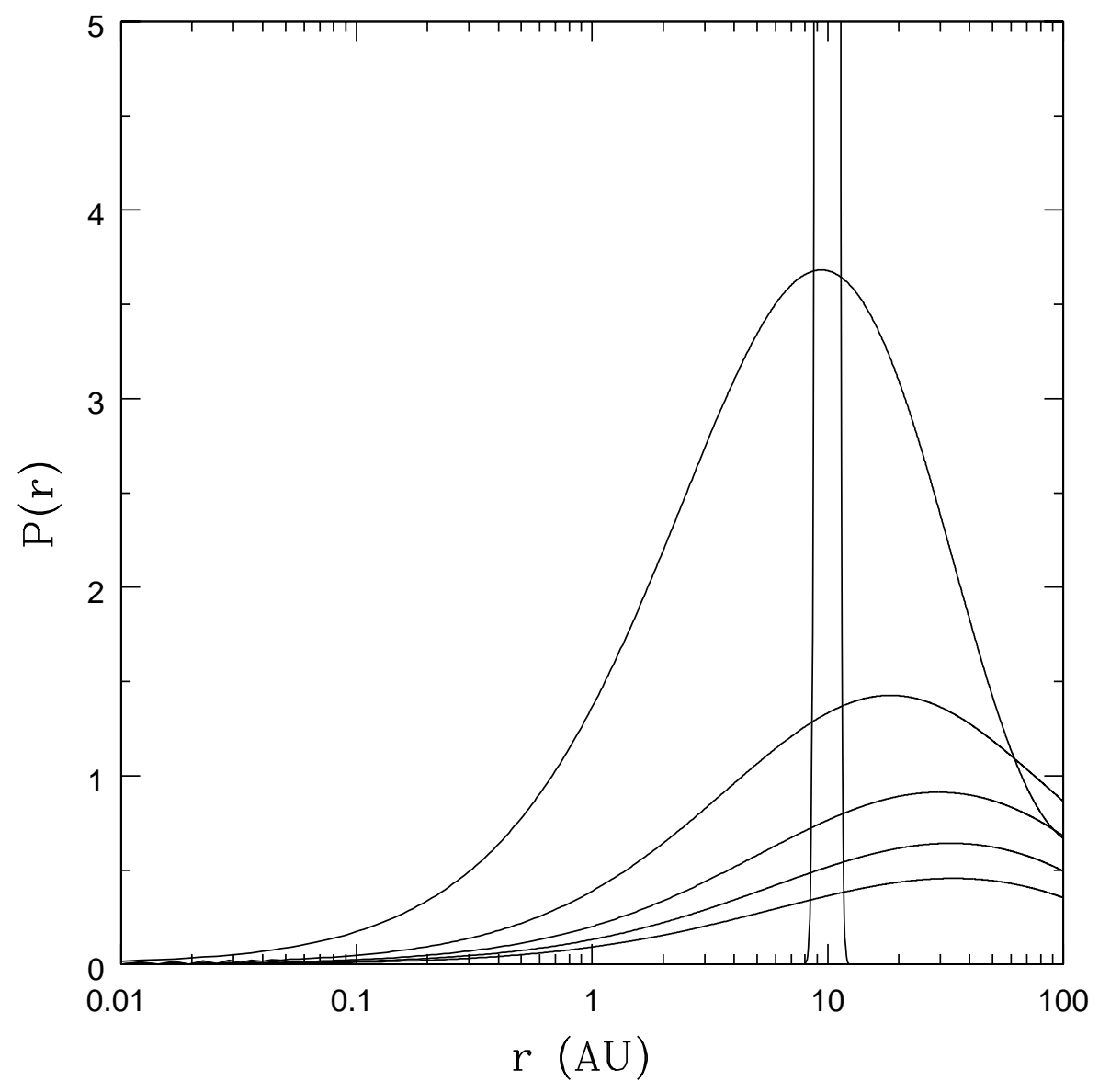

Fig. 1.- Distributions of radial locations of migrating planets from numerical solution to the Fokker-Planck equation. The solution $P$ is the distribution function for the dimensionless angular momentum $x$, so that $P=d p / d x$, but the result is plotted versus radius $r \propto x^{2}$. The solutions are shown at six sampling times: $0,1,2,3,4$, and $5 \mathrm{Myr}$, from top to bottom in the figure. The torque parameters are chosen to be $\gamma=10 \mathrm{Myr}^{-1}$ and $\beta=1 \mathrm{Myr}^{-1}$. Recall that $\gamma \propto m_{P} \Sigma / H^{2}$ (see equation [21]) and that $\beta \propto \Sigma^{2}$ (see equation [22]). 


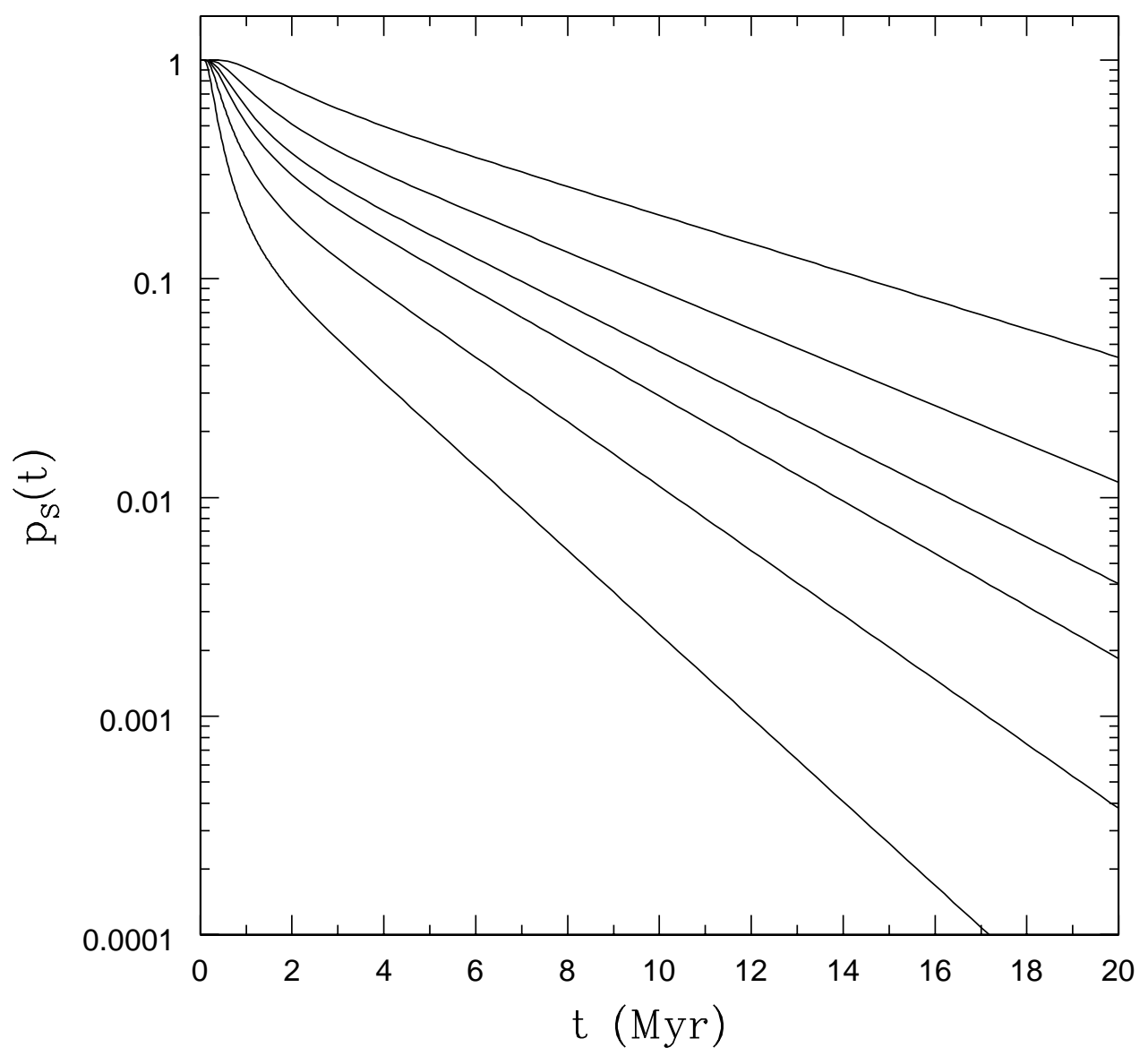

Fig. 2. - Time evolution of the fraction of surviving planets from numerical solution to the FokkerPlanck equation. The curves show the results using varying values of the parameter $\gamma$ that sets the rate of Type I migration relative to the level of turbulence. The values are $\gamma=0,1,3,5,10$, and 20 from top to bottom (in units of $\mathrm{Myr}^{-1}$ ). The Type I migration parameter $\gamma$ scales linearly with both the planetary core mass $m_{P}$ and with the disk surface density $\Sigma$. 
of diffusion, the angular momentum of migrating planets decreases according to $x(t)=x_{0}(1-$ $\left.3 \gamma t / x_{0}^{3}\right)^{1 / 3}$. Using a typical Type I migration rate $\left(\gamma^{-1}=0.1 \mathrm{Myr}\right)$ and the initial conditions of the numerical simulations (where $x_{0} \approx x_{p}=\sqrt{10}$ ), the angular momentum reaches zero (planets are accreted) in time $t_{a c c}=x_{0}^{3} /(3 \gamma)=\sqrt{10} / 3 \approx 1.1$ Myr. For comparison, when turbulent fluctuations are included at the "standard" level (so that $\beta^{-1}=1 \mathrm{Myr}$ ), the survival fraction is $p_{S} \approx 0.36$ at time $t=1 \mathrm{Myr}$ and $p_{S} \approx 0.19$ at time $t=2 \mathrm{Myr}$. The planetary survival fraction falls to $p_{S}=0.10$ at time $t \approx 3.6 \mathrm{Myr}$. Turbulence thus allows planets to survive several times longer than they would otherwise. Nonetheless, in the long time limit, few planets survive: only about 1 percent $\left(p_{S} \approx 0.01\right)$ of the starting population is still present at $t=10 \mathrm{Myr}$.

For the same disk torque parameters used to construct Figure 2, the decay rate $\lambda$ is shown as a function of the parameter $\gamma$ in Figure 3. For the sake of definiteness, the decay rates $\lambda=$ $d \ln p_{S} / d \ln t$ are evaluated at time $t=20 \mathrm{Myr}$. Figure 2 shows that little curvature remains in the survival fractions at times of $20 \mathrm{Myr}$, so that the decay rates have nearly reached their asymptotic values. One should keep in mind, however, that some longer term evolution is possible. In the limit $\gamma=0$, the decay rate approaches the value $\lambda \approx 0.16$, in agreement with the leading order result $\lambda_{1} \approx 0.15$ derived in Section 3.3 using roots of the Bessel function.

As another way to view these systems, we can plot the survival fraction $p_{S}$ as a function of time for fixed Type I torque parameter $\gamma$ and varying values of the diffusion parameter $\beta$. One set of results is shown in Figure 4 for $\gamma=10$ and diffusion parameter in the range $0.1 \leq \beta \leq 10$. For relatively "large" diffusion parameters, corresponding to high levels of turbulence, the survival curves show the same exponential behavior as in Figure 2, For $\beta \approx 0.3$, however, the curves show more structure, and larger fractions of the planetary population survive. For even smaller values of the diffusion parameter (not shown in the Figure), turbulence has little effect, and steady disk torques sweep (almost) the entire population of planets into the star on the Type I migration timescale. This behavior suggests that for a fixed value of $\gamma$, there exists an optimum value of the diffusion parameter $\beta$ that maximizes the number of surviving planets. This optimum value depends on the time of observation and is taken up in Section 4.2.

All of the results shown thus far correspond to the same initial distribution of angular momentum, i.e., a narrow gaussian centered on the angular momentum appropriate for a circular orbit at $r_{0}=10 \mathrm{AU}$. We expect the planetary survival rate depend on the starting location. As outlined in Section 2, for typical torque parameters, the time scale for inward Type I migration and that for turbulent diffusion are comparable for radii near 10 AU. For smaller radii, Type I torques are dominant, and fewer planets should survive. For larger radii, turbulence dominates. To study this issue, we have performed a series of simulations in which the starting location is a narrow gaussian centered on an angular momentum value corresponding to a range of radial locations from 1 AU to the outer disk edge. The results are shown in Figure 5 for four sampling times $(1,3,5$, and 10 Myr). Notice that the four curves display a sharp corner near $r_{0} \sim 10$ AU. For smaller radii, the survival fraction $p_{S}$ drops precipitously. For larger radii, the fraction $p_{S}$ is a fairly flat function of radius at a given sampling time. 


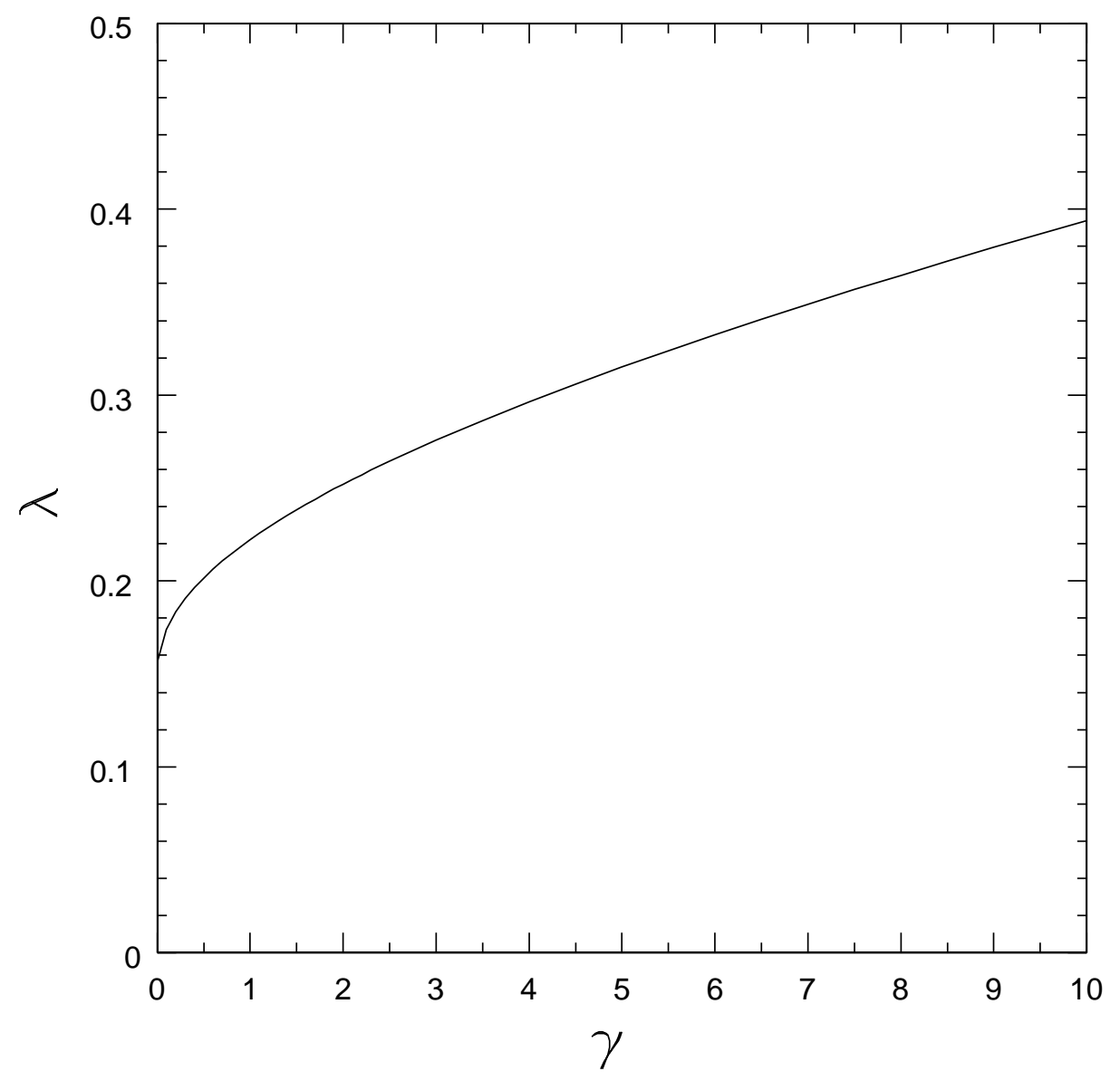

Fig. 3.- Exponential decay rate $\lambda$ for planet survival as a function of Type I migration parameter $\gamma$ for fixed diffusion parameter (here $\beta=1$ ), where all quantities are given in units of $\mathrm{Myr}^{-1}$. The decay rates are evaluated from the numerical solutions at an evolution time of $20 \mathrm{Myr}$. 


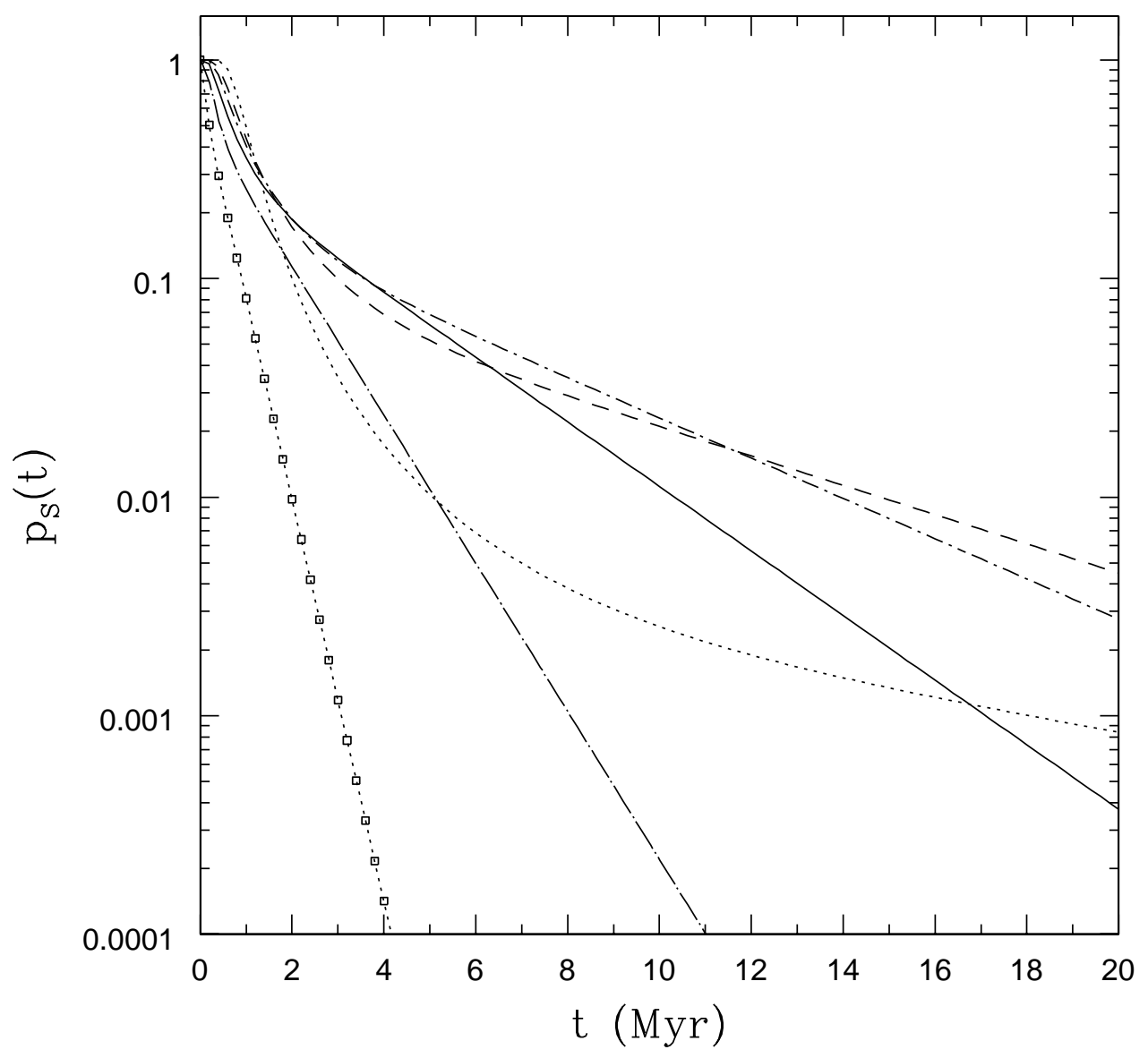

Fig. 4. - Time evolution of the fraction of surviving planets from the Fokker-Planck equation using varying values of the diffusion parameter $\beta$. The curves correspond to values of $\beta=0.1$ (dots), $\beta$ $=0.3$ (dashes), $\beta=0.5$ (dot-dashes), $\beta=1$ (solid), $\beta=3$ (dot-long-dashes), and $\beta=10$ (dots marked by open squares). The diffusion parameters are given in units of $\mathrm{Myr}^{-1}$. 


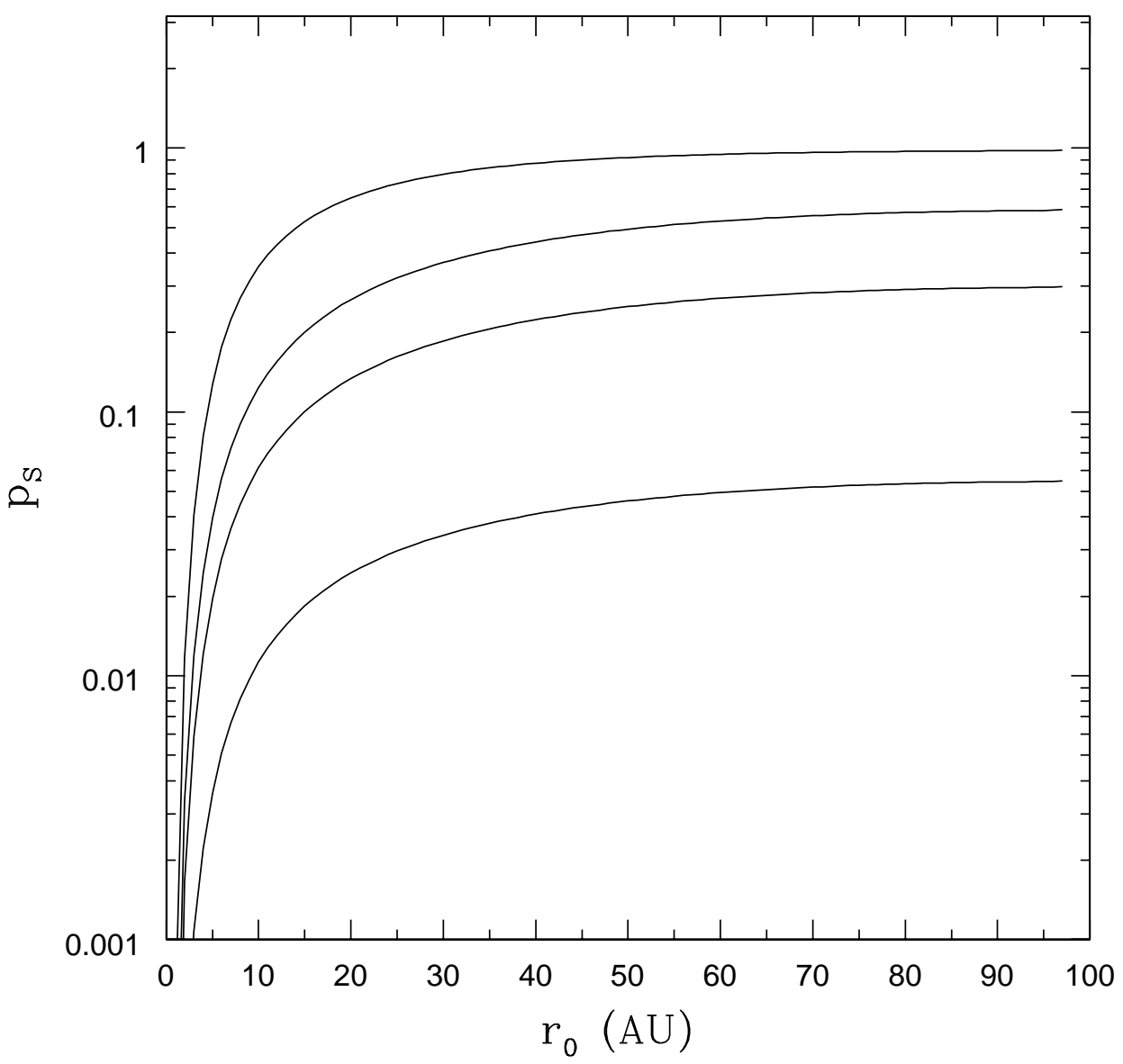

Fig. 5.- Planet survival fraction as a function of starting location. In each of these simulations, the initial distribution of angular momenta is taken to be a narrow gaussian centered on a value given by $x_{0}=\left[r_{0} /(1 \mathrm{AU})\right]^{1 / 2}$. The fraction of surviving planets is plotted as function of $r_{0}$ for four sampling times: $t=1 \mathrm{Myr}, 3 \mathrm{Myr}, 5 \mathrm{Myr}$, and $10 \mathrm{Myr}$ (from top to bottom in the figure). 


\subsection{Optimization of Survival Probability}

For a given Type I migration rate and a given time, there exists an optimum value of the diffusion constant that provides the greatest number (fraction) of surviving planets. This claim can be seen as follows: In the limit of no diffusion $D \rightarrow 0$, the planets all migrate inward. For times greater than the Type I migration time, essentially all of the planets are accreted by the central star, and the number of surviving planets approaches zero. In the opposite limit where the diffusion constant is large, the random walk in angular momentum introduced by the diffusion process would lead to crossings of the origin (where $j \rightarrow 0, r \rightarrow 0$, and accretion takes place) in only a few steps. Given the one-way barrier at the stellar surface, the fraction of surviving planets also vanishes in the limit of large $D$. As a result, an optimum value of the diffusion constant can occur in the intermediate regime.

Figure 6 shows the results of numerically exploring this optimization problem. At fixed sampling times, the fraction of surviving planets is shown as a function of the diffusion constant for a fixed value of the Type I migration torque $\left(\gamma=10 \mathrm{Myr}^{-1}\right)$. At early times (the uppermost curve in the figure at $t=1 \mathrm{Myr}$ ), the Type I migration process has not had time to remove all of the planets, and the result of increasing the diffusion constant is to decrease the number of surviving bodies. At all later times shown, however, a maximum appears in the survival fraction at intermediate values of the diffusion constant. Note that this maximum occurs for values of the diffusion parameter near those expected from "typical" turbulent torques, although a wide range of such parameters are possible.

The optimum value of the diffusion parameter depends on the other properties of the system: Here we have used the expected value of the Type I migration parameter $\gamma=10 \mathrm{Myr}^{-1}$ and used initial conditions where the planetary cores are formed near $r=10$ AU. However, notice that with the general form of the Fokker-Planck equation (20), one can absorb the parameter $\gamma$ into the definition of time, so that the results depend only on the ratio $\beta / \gamma=Q_{\mathrm{m}}$ (see equation [15]). Further, $Q_{\mathrm{m}}$ depends on the physical properties of the systems according to $Q_{\mathrm{m}} \propto \Sigma H^{2} / m_{P}$.

To illustrate this optimizing behavior, we consider the simplified, self-similar version of the problem developed in Section 3.4. Specifically, we use the self-similar solution of equation (51) as a model for the dynamics. This treatment does not include the outer disk boundary, and hence overestimates the survival probability. On the other hand, it provides an analytic understanding of how the parameter space of Type I torque strength (given here by $\langle\gamma\rangle$ ) and diffusion constant $\beta$ contains a local maximum in the fraction of surviving planetary cores.

Given the normalized solution of equation (52) for the survival fraction $p_{S}$ as a function of time, we can find the optimum value of the diffusion parameter $\beta$ for fixed time $t$ and migration parameter $\langle\gamma\rangle$. The extremal value occurs where $d p_{S} / d \beta=0$, which implies the constraint

$$
\langle\gamma\rangle \ln [1+\beta t / \sigma]=\frac{\beta t / \sigma}{1+\beta t / \sigma}(\beta+\langle\gamma\rangle)
$$




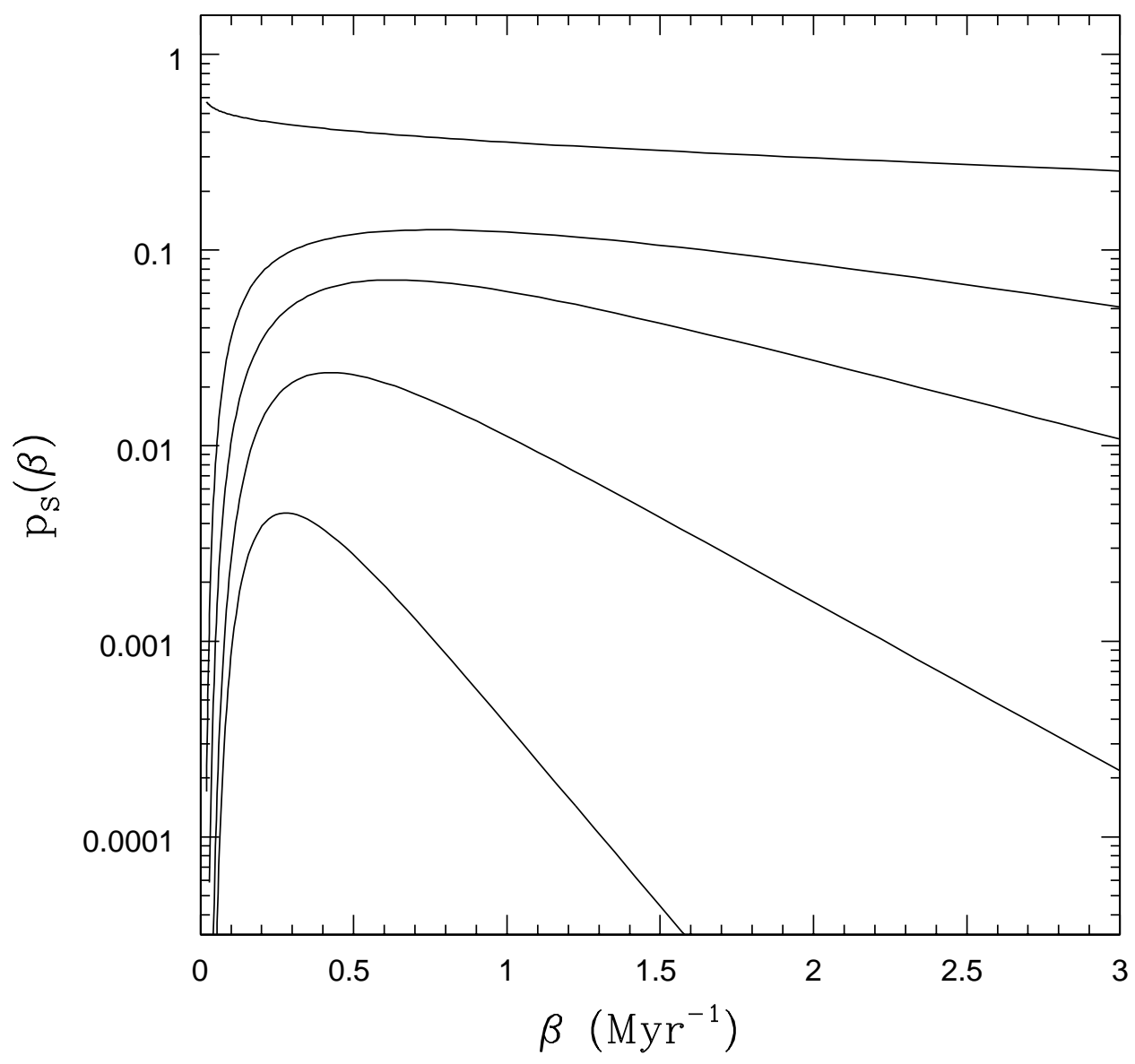

Fig. 6. - Total survival fraction as a function of the diffusion parameter $\beta$ (the value of the diffusion constant at $r=1 \mathrm{AU}$ in units of $\mathrm{Myr}^{-1}$ ). The Type I migration parameter $\gamma$ is kept constant at a value of $10 \mathrm{Myr}^{-1}$. The curves shown correspond to times of $1 \mathrm{Myr}$ (top), $3 \mathrm{Myr}, 5 \mathrm{Myr}, 10 \mathrm{Myr}$, and $20 \mathrm{Myr}$ (bottom). The initial distribution of angular momentum for this set of simulations was a narrow gaussian centered at $x=\sqrt{10}$, i.e., $r=10 \mathrm{AU}$. 
Equation (54) has a solution provided that the parameter $\alpha \equiv\langle\gamma\rangle t / \sigma \geq 2$ (at the point of equality, the solution corresponds to $\beta=0$ ). When this condition is met, the solution to equation (54) determines the optimum value of the diffusion constant for which the maximum fraction of planetary cores survive.

The resulting optimized survival fraction is shown in Figure 7 as a function of the parameter $\alpha=\langle\gamma\rangle t / \sigma$. Notice that for small values of $\alpha<2$ the optimization condition (54) has no solution. For the regime where $\alpha=\langle\gamma\rangle t / \sigma<2$, the fraction of planetary cores is a decreasing function of the diffusion constant $\beta$; in this regime, the Type I migration has not had time to completely deplete the planetary population, so that increasing the diffusion constant leads to loss of planets rather than helping to save them. For the same choice of parameters used above $\left(\langle\gamma\rangle=0.3 \mathrm{Myr}^{-1}, \sigma=\right.$ 1 , and time $t=10 \mathrm{Myr}$ ), the optimal survival fraction is about $p_{S} \approx 0.064$ (compared to the value of $p_{S} \approx 0.044$ obtained previously with $\beta=1 \mathrm{Myr}^{-1}$ ).

\section{3. $\quad$ Long Time Limit}

The most important outcome of the diffusion process considered herein is the fraction of surviving planets and their distribution of positions (given by their angular momentum in this formulation). These quantities are determined by the solutions to the Fokker-Planck equation. Although one can find numerical solutions (see above), analytic or simplified descriptions can greatly add to our understanding of the issues. The analytic treatment developed in Section 3.3, where the Type I migration torques were turned off, can be generalized to provide a full solution. For the full problem, including the Type I migration term, one can separate variables and find an analogous series solution. In this case, however, the spatial eigenfunctions $F(x)$ are hypergeometric functions (AS), rather than Bessel functions, and hence are cumbersome to work with. Fortunately, in the long time limit, the problem simplifies greatly. In this asymptotic limit, only the lowest order term in the expansion survives, and the distribution is determined by the solution to the following eigenvalue problem

$$
\beta \frac{d^{2}}{d x^{2}}\left(x^{b} F\right)+\gamma \frac{d}{d x}\left(\frac{F}{x^{a}}\right)+\lambda_{1} F=0,
$$

where $\lambda_{1}$ is the lowest order eigenvalue and $F(x)$ is the corresponding eigenfunction. Note that we can absorb one of the parameters. For example, we can divide equation (55) by $\gamma$ and work in terms of a relative diffusion constant $\widetilde{\beta}=\beta / \gamma$. The scaled eigenvalue $\widetilde{\lambda}=\lambda_{1} / \gamma$ will then be dimensionless.

Figure 8 shows the distributions calculated from our numerical treatment of the Fokker-Planck equation in the long time limit. In this case, the standard form of the Fokker-Planck equation (with $a=2, b=1, \beta=1 \mathrm{Myr}^{-1}$, and $\gamma=10 \mathrm{Myr}^{-1}$ ) was integrated out to $100 \mathrm{Myr}$. The five solid curves shown in the figure correspond to times of 10, 20, 30, 40, and $50 \mathrm{Myr}$, from top to bottom in the figure. Notice that the five curves are nearly parallel to each other and exhibit nearly equal spacing. As a result, the distributions have reached an asymptotic form, and are decreasing in amplitude 


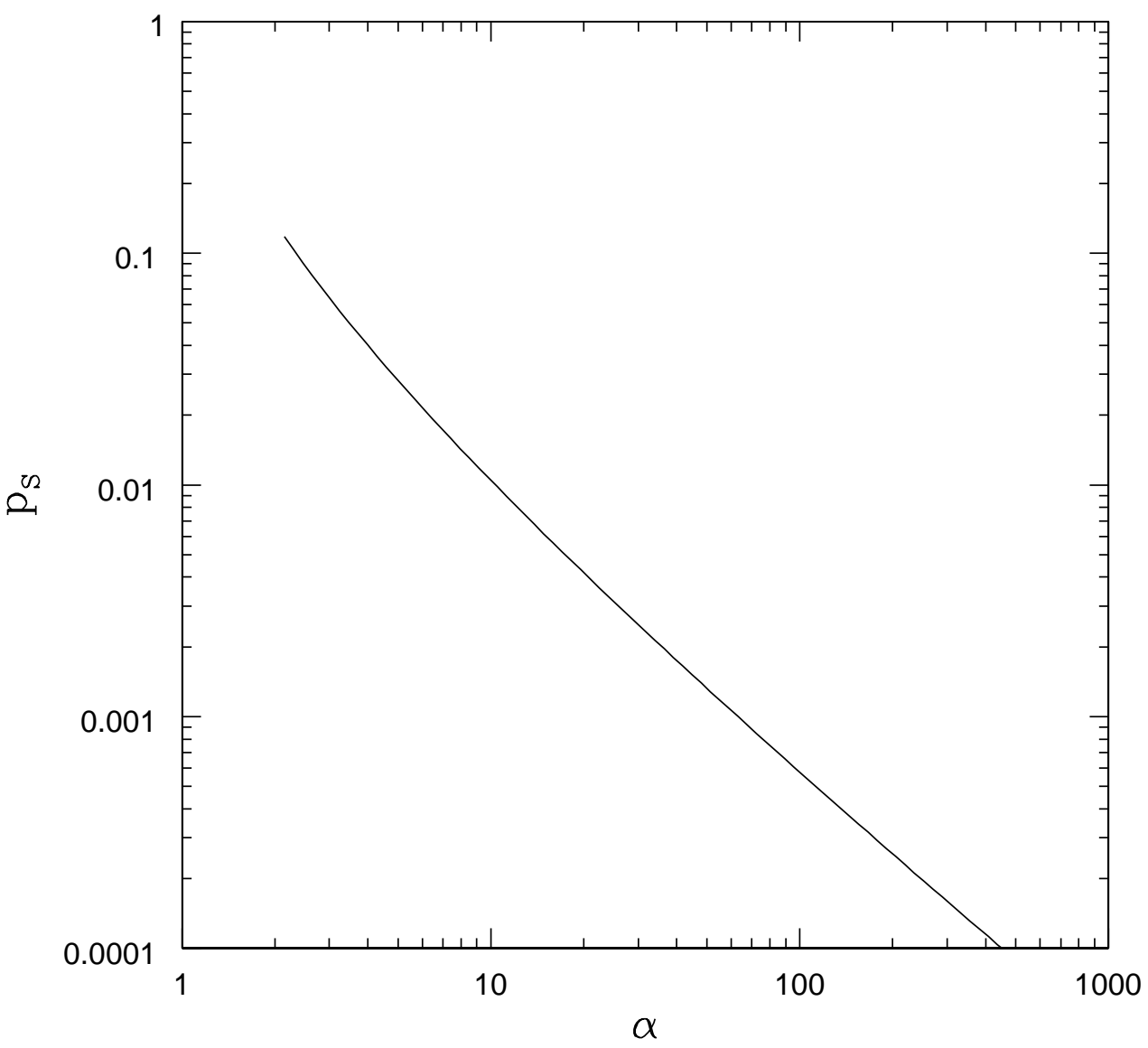

Fig. 7.- Fraction $p_{S}$ of surviving planets as a function of $\alpha=\langle\gamma\rangle t / \sigma$, where the diffusion constant has been optimized using the self-similar solution of Section 3.4. Notice that for $\alpha<2$, no optimizing solution exists; in this regime, diffusion acts to reduce the number of surviving planets. For $\alpha>2$, diffusion acts to increase the probability $p_{S}$ of planetary survival. 


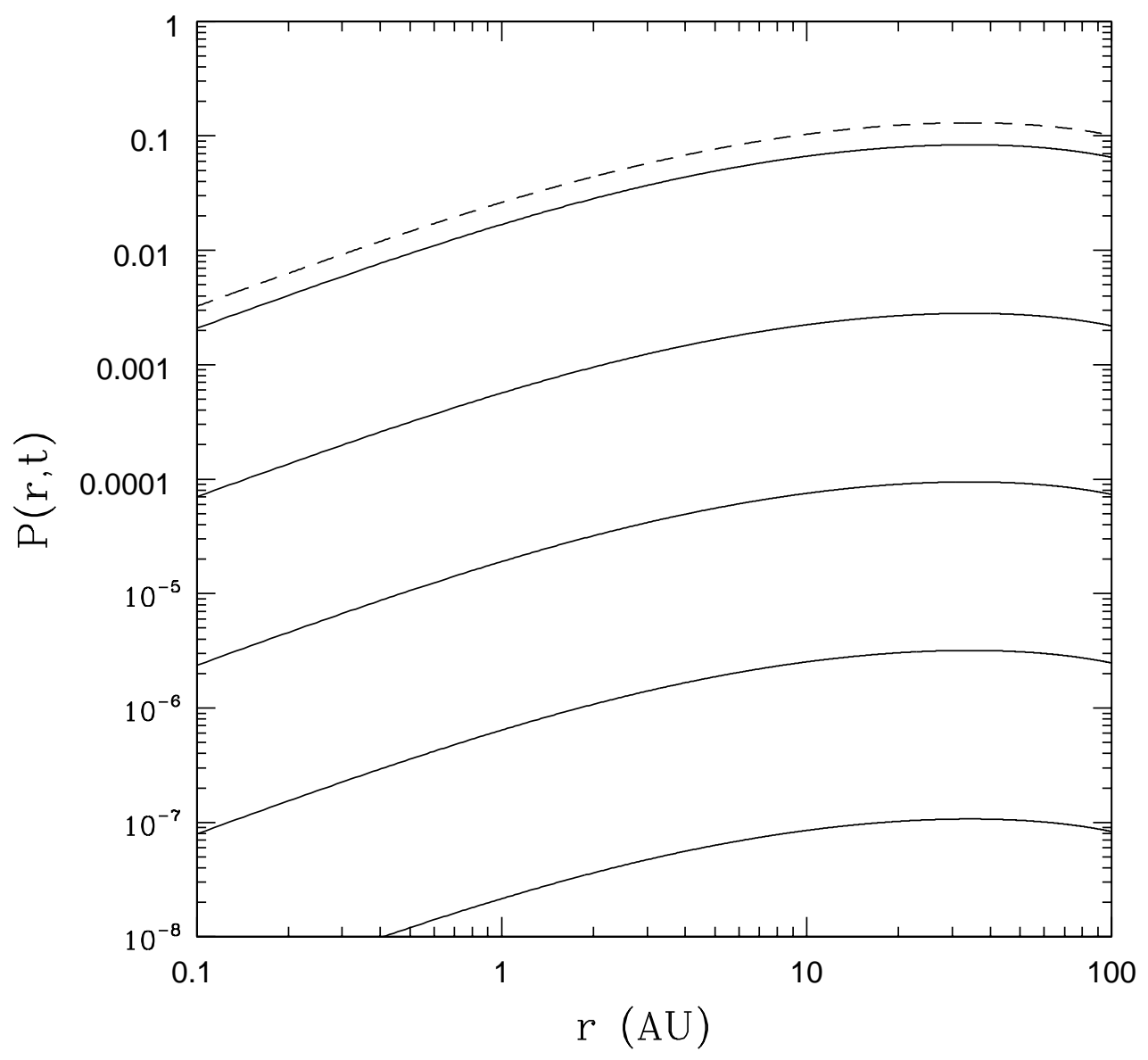

Fig. 8.- Distributions $P(r, t)$ in the long time limit. The solid curves show the distributions resulting from numerically integrating the standard form of the Fokker-Planck equation at five times: $10 \mathrm{Myr}$ (top curve), $20 \mathrm{Myr}, 30 \mathrm{Myr}, 40 \mathrm{Myr}$, and $50 \mathrm{Myr}$ (bottom curve). The lowest order eigenfunction from equation (55) is plotted as a dashed curve just above the uppermost solid curve. This eigenfunction has almost exactly the same shape as the distributions predicted by the FokkerPlanck equation (the eigenfunction must be offset from the numerically determined distribution to be visible in the plot). 
with a well-defined decay rate. The lowest order eigenfunction calculated from equation (55) is also shown as a dashed curve, just above the uppermost solid curve. If this eigenfunction is plotted with the same normalization as the distributions resulting from the Fokker-Planck equation, the functions are indistinguishable. This figure thus demonstrates that the lowest order eigenfunction provides a good description of the solution in the long time limit. Furthermore, this limit is reached on a time scale less than $10 \mathrm{Myr}$.

Given that the solutions can be described by the lowest order eigenfunctions, we can estimate the probable locations for surviving planetary cores. For given values of the Type I migration parameter $\gamma$ and the diffusion parameter $\beta$, we can find the eigenvalues and corresponding eigenfunctions for equation (55). The results are shown in Figure 9 for a fixed value of the Type I migration parameter $\gamma$ and for three values of the diffusion parameter: $\beta / \gamma=0.01,0.1$, and 1 ; note that only the ratio $\beta / \gamma=Q_{\mathrm{m}}$ (see equation [15]) is needed to determine the form of the solutions. As expected, these probability distributions peak in the outer part of the disk. As the diffusion parameter increases, the distributions become wider, and hence have more support at smaller radii. The three distributions shown in Figure 9 are normalized to have the same integrated value. For larger $\beta$ and fixed $\gamma$, however, the survival probability is a decreasing function of the diffusion parameter in this regime (see Figure 6 ).

This procedure also specifies the eigenvalues, which in turn determine the decay rates for planet survival in the long term. For fixed Type I migration parameter $\gamma=10$, and for diffusion parameters $\beta=0.1,1$, and 10 , the lowest order eigenvalues are $\lambda_{1} \approx 0.1098,0.3400$, and 2.022 , respectively (where all quantities are in units of $\mathrm{Myr}^{-1}$ ). For diffusion parameter $\beta=1$, corresponding to the expected center of parameter space, this eigenvalue compares favorably with those calculated from numerical solutions to the Fokker-Planck equation (see Figures 2 and 3 ).

\subsection{Time Dependent Torque Parameters}

Both the Type I migration torque and the stochastic torques due to turbulent forcing depend on the surface density of the disk. Since the disk mass is expected to be a decreasing function of time, the normalization of the disk surface density will, in general, be time dependent. To gain some understanding of how this time dependence affects the migration problem considered herein, we assume that the disk surface density maintains the same power-law form, but the disk mass decreases with time. Toward this end, we introduce a normalization function $s(t)$ such that the disk mass is given by $M_{d}(t)=M_{d}(0) s(t)$. Although the form of $s(t)$ is not known, observations show that circumstellar disks lose their mass on time scales of order 3 - 10 Myr (Haisch et al. 2001, Hernández et al. 2007, Hillenbrand 2008). More specifically, the observational sample shows that about half of the stars lose their disks by age $\sim 3 \mathrm{Myr}$, and that only about $1 / e \sim 1 / 3$ of the disks remain at $5 \mathrm{Myr}$. For the sake of definiteness, we use a simple exponential form for $s(t)$, i.e.,

$$
s(t)=\exp \left[-t / t_{0}\right]
$$




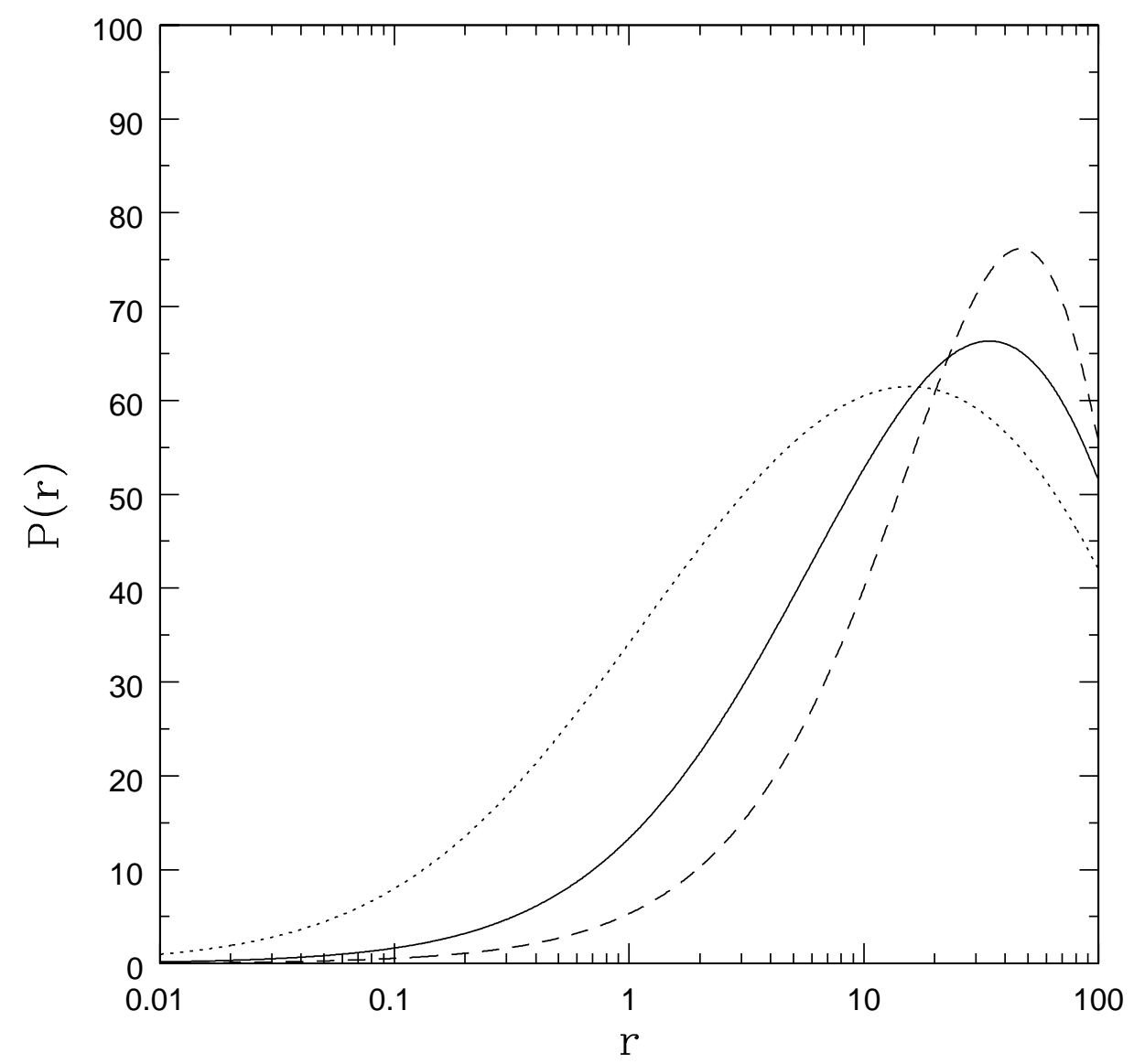

Fig. 9.- Eigenfunctions for the lowest order mode solution to the Fokker-Planck equation. In the long time limit, these functions provide the distribution of angular momentum, and hence radial position, for surviving planetary cores. The three curves shown here correspond to a fixed Type I migration parameter $\gamma$ and varying values of the diffusion parameter given by $\beta / \gamma=0.01$ (dashed curve), 0.1 (solid curve), and 1 (dotted curve). As shown, the three eigenfunctions are normalized to the same (arbitrary) value. 
where we expect the time scale $t_{0}=1-10 \mathrm{Myr}$.

Next we note that the Type I migration torque is proportional to the surface density $\Sigma(r)$, whereas the effective diffusion constant from the turbulent torques scales like $\Sigma^{2}$. When the FokkerPlanck equation is modified to include this time dependence, it takes the form

$$
\frac{\partial P}{\partial t}=\gamma s(t) \frac{\partial}{\partial x}\left(\frac{P}{x^{2}}\right)+\beta s^{2}(t) \frac{\partial^{2}}{\partial x^{2}}(x P)
$$

where we have used the standard radial dependence of the surface density and temperature (and the standard $x$-dependence of the torques). Note that $\gamma$ and $\beta$ are defined by equation (19).

In this formulation, the two terms on the right hand side of the Fokker-Planck equation (57) display different types of time dependence. For purposes of illustration, we can consider one term at a time. For the case in which only one of the torque terms is operational, we can define a new time variable $\tau$ according to $d \tau_{1}=s(t) d t$ or $d \tau_{2}=s^{2}(t) d t$. With the former substitution, the Type I migration dynamics becomes the same as that considered in Section 3.2, with the time $t$ replaced by $\tau_{1}$. Similarly, the diffusion dynamics becomes the same as that considered in Section 3.2, with $t$ replaced by $\tau_{2}$. With time dependence surface density, however, the effective time variables $\tau_{j}(t)$ reach finite values in the limit $t \rightarrow \infty$, i.e.,

$$
\tau_{1}(t)=t_{0}\left[1-\mathrm{e}^{-t / t_{0}}\right] \rightarrow t_{0} \quad \text { and } \quad \tau_{2}(t)=\frac{t_{0}}{2}\left[1-\mathrm{e}^{-2 t / t_{0}}\right] \rightarrow \frac{t_{0}}{2} .
$$

Thus, the net effect of decreasing disk mass is to limit the operation of Type I torques to an effective time of $t_{0}$, and to limit the operation of diffusion to an effective time of $t_{0} / 2$. On one hand, the result that decreasing disk mass implies a finite time for torques to act is expected. Further, the effective time depends on the function $s(t)$ that describes the time dependence. On the other hand, the two types of torques depend on disk mass - and hence on $s(t)$ - in different ways and hence have different effective duty cycles. For exponential decay in disk mass, Type I migration torques act over a time span that is effectively twice as long as that of turbulent diffusion.

For a given version of the Fokker-Planck equation, and a given time dependence $s(t)$ for the surface density and disk mass, we can find numerical solutions. The result is shown in Figure 10 for the standard choice of power-law disk parameters. The time dependence of the disk mass has the exponential form given by equation (56) with different values of the decay time: $t_{0}=1,3,10$, 30 , and the limit of constant disk mass $t_{0} \rightarrow \infty$. For each case, the survival fraction is shown as a function of time. For finite $t_{0}$, both types of torques become ineffective over a sufficiently long span of time, and the survival fraction asymptotically approaches a constant value. Moreover, for expected values of the disk lifetime, these asymptotic values are $p_{S} \approx 0.04-0.16$.

The Type I torque parameter also depends on the mass of the growing planetary core, and this time dependence can also be included. Here we present a simple working model to illustrate the type of behavior introduced by this time dependence. At early times, when the planetary mass $m_{P}<10 M_{\oplus}$, the Type I torque parameter depends linearly on the mass (see equation [21]). For 


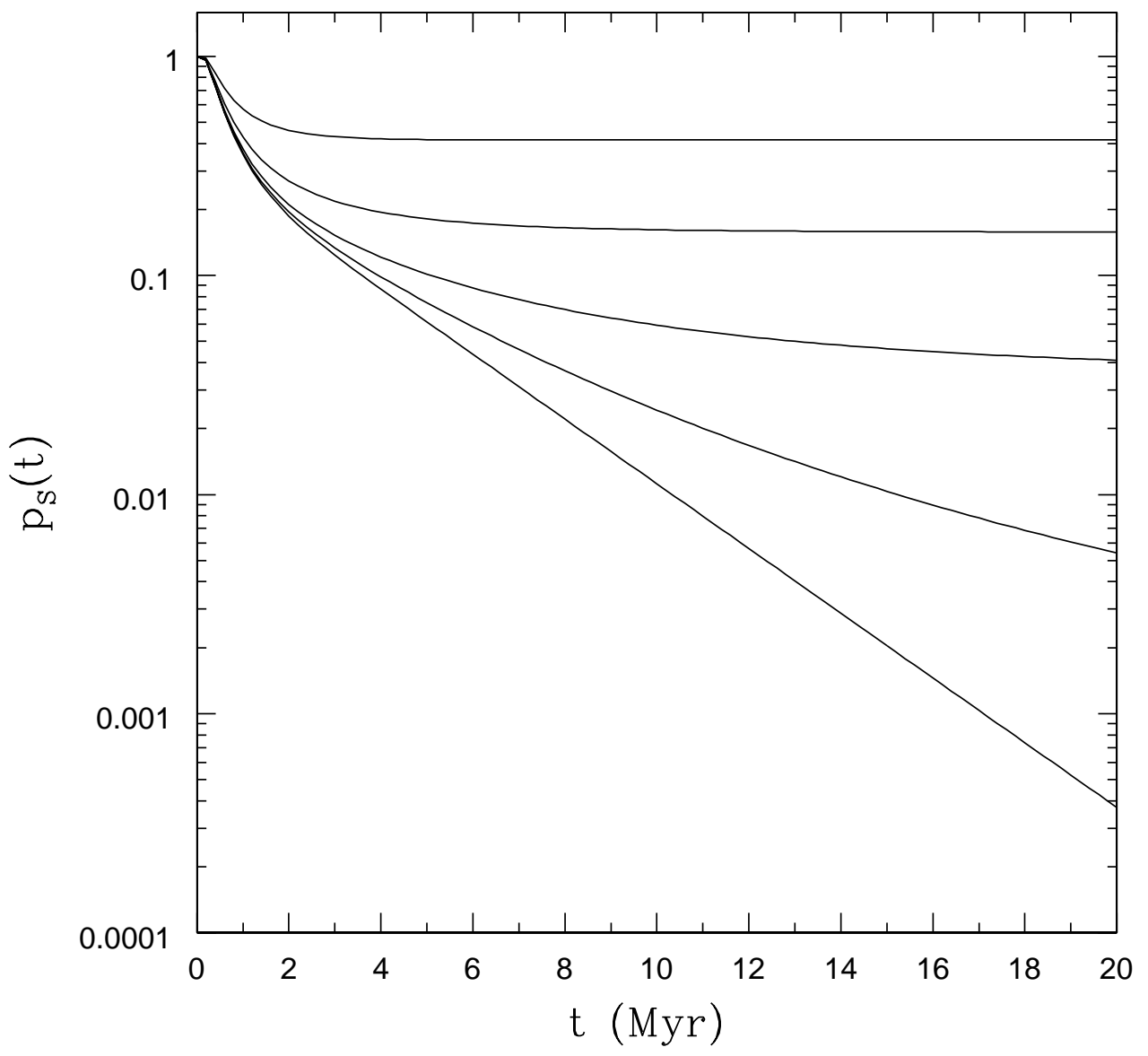

Fig. 10.- Survival fraction as a function of time for systems where the disk mass decreases with time. The curves shown here correspond to different exponential time constants for disk mass evolution: $t_{0}=1,3,10,30$, and the limit $t_{0} \rightarrow \infty$ (from top to bottom). 


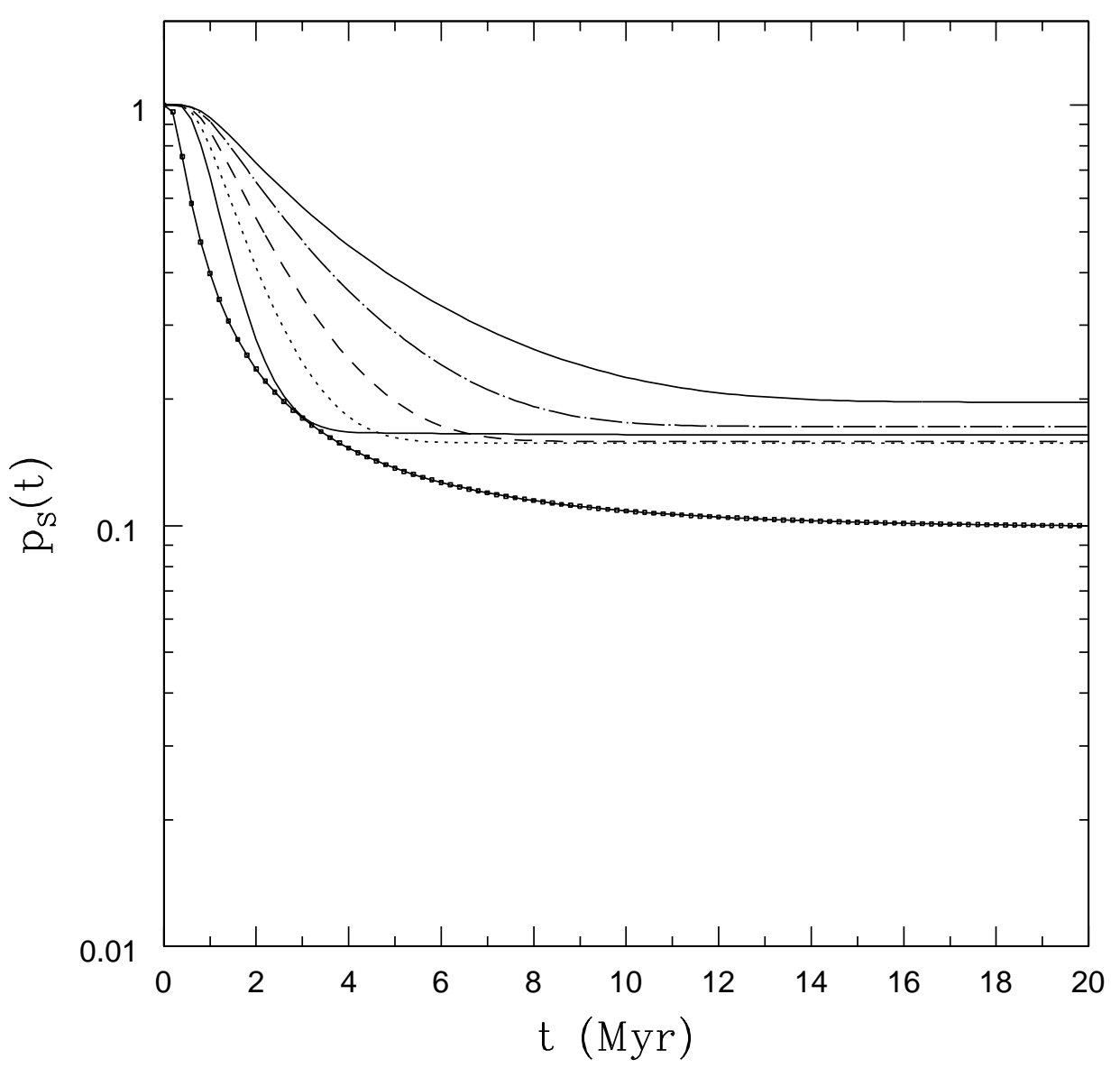

Fig. 11. - Survival fraction $p_{S}$ as a function of time for systems where the Type I torque parameter is time dependent and the disk mass decreases with time. The torque parameter is taken to have the form given by equation (59), where the planetary mass $m_{P}$ increases with time according to $m_{P}=m_{1}(t / 1 \mathrm{Myr})^{3}$. The disk mass decreases with time constant $t_{0}=5 \mathrm{Myr}$. The curves shown here correspond to $m_{1} / M_{\oplus}=1$ (lower solid curve), 0.3 (dotted curve), 0.01 (dashed curve), 0.003 (dot-dashed curve), and 0.001 (upper solid curve). The lower curve marked by open squares shows the result with a constant value $\gamma=10 \mathrm{Myr}^{-1}$. 
larger masses $m_{P} \sim 30-100 M_{\oplus}$, however, the planet clears a gap in the disk, and the migration torques become much smaller. We represent this general trend by taking the torque parameter $\gamma$ to have the simple form

$$
\gamma=\Gamma\left(m_{P} / M_{\oplus}\right) \exp \left[-m_{P} / m_{C}\right]
$$

The function $\gamma\left(m_{P}\right)$ attains its maximum value at $m_{P}=m_{C}$. For the sake of definiteness, here we take $m_{C}=10 M_{\oplus}$. The corresponding maximum value is then given by $\gamma=10 \Gamma / e$. If we use $\Gamma=10$ $\mathrm{Myr}^{-1}$, the maximum value of $\gamma \approx 30 \mathrm{Myr}^{-1}$, a typical Type I migration parameter expected for $m_{P} \approx 12 M_{\oplus}$ (see equation [21]). At the expected gap clearing mass of $m_{P} \sim 30 M_{\oplus}$, the Type I migration parameter $\gamma \sim 15 \mathrm{Myr}^{-1}$, and it decreases rapidly with further increases in $m_{P}$.

Next we need to specify the mass of the planetary core as a function of time. At relatively small masses, the core grows with accretion rate $\dot{M} \propto R^{2}$, where $R$ is the radius of the planet (e.g., Lissauer \& Stevenson 2007). For constant planetary density, the mass grows with time according to $m_{P} \propto t^{3}$. At later times, when the planet is large enough for gravitational focusing to become important, the accretion rate approaches the form $\dot{M} \propto R^{4}$ and the mass increases rapidly. Once the planetary core reaches this phase, however, it becomes large enough to clear a gap and the Type I torques are significantly less important. We thus concentrate on the early phase, and hence allow the planetary mass to grow according to $m_{P}=m_{1}(t / 1 \mathrm{Myr})^{3}$. The parameter $m_{1}$ depends on the surface density of solids in the disk and the radius of core formation. Here we take this mass scale to lie in the range $m_{1} / M_{\oplus}=0.01-1$. For this range, the corresponding time required for a growing planetary core to reach the threshold value of $m_{P}=10 M_{\oplus}$ is $t \sim 2-10 \mathrm{Myr}$.

Using the above time dependence for the Type I torque parameter, the Fokker-Planck equation can be integrated as before, also including the decrease in disk surface density through the function $s(t)$. The result is shown in Figure 11 for a disk evolutionary timescale of $t_{0}=5 \mathrm{Myr}$, and for $m_{1}$ $=0.01-1 M_{\oplus}$. The curve for $\gamma=10 \mathrm{Myr}^{-1}=$ constant is also shown (for the same timescale $t_{0}$ ). In the scenario with time dependent planetary mass, the Type I torque parameter is smaller than our assumed constant value at early times, but larger at later times. To leading order, the time dependence tends to cancel out. Since planetary core masses grow rapidly, however, the systems spend more time with lower torque parameter values, so that the inclusion of this time dependence allows more planetary cores to survive (see Figure 11). We can understand this result by defining an effective duty cycle $\tau_{3}$ for the Type I torques, analogous to those in equation (58), by including both the time evolution of the planetary mass and the disk surface density. For the parameters used here, this time scale lies in the range $\tau_{3} \approx 4.2-5.6 \mathrm{Myr}$. These timescales are close to that for disk evolution only, $\tau_{1}=t_{0}=5 \mathrm{Myr}$, indicating that the smaller values of $\gamma$ at early times nearly cancel the larger values at later times. This (approximate) cancellation is reflected in the survival fractions, which are confined to the range $p_{S} \approx 0.1-0.2$ for the cases shown in Figure [11, Of course, disk systems can display a wide range of parameters, so that smaller survival fractions can also be realized. 


\section{CONCLUSION}

This work reinforces and extends results obtained in previous studies (LSA, NP, JGM): Turbulence transforms Type I migration from a steady inward progression into a diffusive process. Turbulence thus allows some fraction of the population of planetary cores to survive beyond the Type I migration timescale. However, the outcome of any particular migration episode is uncertain because of extreme sensitivity to initial conditions - due to chaos - so that the results must be described in terms of probability distributions (see Figures 1, 8, 9, and 12). This survival problem, where steady inward migration is coupled to stochastic behavior, and where the torques associated with both effects can vary with time, allows for a rich diversity of behavior. A more specific description of our results is given below (Section 5.1) along with a discussion of their implications and limitations (Section 5.2).

\subsection{Summary of Results}

Stochastically driven diffusion, due to turbulent torques, can act to save planetary cores from accretion due to Type I migration. For torque strengths near the center of the expected range of parameter space (specifically, $\gamma=10 \mathrm{Myr}^{-1}, \beta=1 \mathrm{Myr}^{-1}$, and active disk lifetime $t=3 \mathrm{Myr}$ ), and for planets starting near $r=10 \mathrm{AU}$, the survival fraction $p_{S} \approx 0.1$ (see Figures 2, 4, and 6). Note that this "lifetime" can be the time required for the planetary core to reach the threshold required for gap clearing, so that migration slows down. For longer timescales, the fraction of surviving bodies is much smaller. For the same torque parameters, the survival fraction $p_{S} \approx 0.01$ at $t=10$ Myr and $p_{S} \approx 0.0004$ at $t=20 \mathrm{Myr}$. Keep in mind that these survival fractions are modified when the torque parameters exhibit time dependence (see below).

The outer boundary condition in the disk plays an important role in determining the fraction of surviving planets. A finite disk edge causes the fraction of surviving planets $p_{S}(t)$ to experience exponential decay (see Figure 2, Sections 3.3 and 4.3), whereas a disk with infinite extent displays power-law decay (see Section 3.4 and JGM). In most cases of interest, the expected disk outer radius $\left(r_{\text {disk }} \sim 30-100 \mathrm{AU}\right)$ is small enough that planets can diffuse to the outer boundary during the active disk lifetime, so that edge effects are important and exponential decay is realized. Typical

decay rates lie in the range $\lambda=0.1-0.5 \mathrm{Myr}^{-1}$, and are found from both analytic calculations (Sections 3.3 and 4.3) and numerical simulations (Figures 2 and 31).

The probability of planet survival is sensitive to the initial conditions. The most favorable locations for forming planetary cores lie just outside the snow-line in circumstellar disks, i.e., in the radial range $5-10$ AU. For typical torque parameters, this regime also marks the boundary between the outer disk, where turbulent torques dominate, and the inner disk, where inward Type I migration torques dominate. As a result, planetary cores starting their migration within this annulus are particularly sensitive to the specifics of their evolution. Planets forming at somewhat larger radii are much more likely to survive, whereas planets that form at smaller radii have little 
chance of survival (Figure 5).

For a given value of the Type I inward migration torque amplitude, there exists an optimum value of the diffusion constant that leads to the maximum number of surviving planets. This extremum depends on the effective disk lifetime. For sufficiently short lifetimes (shorter than the nominal Type I migration time), diffusion acts to reduce the fraction of surviving planets and the optimum value of the diffusion constant is zero. For longer disk lifetimes, diffusion acts to save planets, and a maximum develops in the survival curve (see Figure 6). The optimum value of the diffusion constant corresponds to an optimum level of turbulence. Furthermore, this optimum level of turbulence is relatively near that found in previous MHD simulations (LSA, NP, Nelson 2005). The existence of an optimal value of the diffusion constant can be derived analytically using the self-similar limiting form of the problem (see Section 4.2, Figure 7, and equations [52, 54]).

In the long time limit, the distributions of angular momenta for surviving planets approach a well-defined form (see Figure 8), with the amplitude (normalization) decreasing at a well-defined decay rate. The form of this asymptotic distribution is given by the lowest order eigenfunction of the spatial part of the Fokker-Planck equation (see Figure 9), and the decay rate is given by the corresponding eigenvalue (see equation [55]). The distribution of surviving planets peaks in the outer disk and provides the initial conditions for the later stages of planetary growth.

The time dependence of the disk mass and surface density leads to corresponding time dependence in the torque parameters, and can be incorporated into this formulation of the diffusion problem (Section 4.4). Because the two types of torques depend on different powers of the surface density, the effective duty cycle of the Type I migration torque is longer than that due to turbulence. When the time dependence of the disk surface density is included, the survival probability of planets approaches a well-defined asymptotic value (Figure 10) that depends on the disk evolutionary timescale $t_{0}$ (equation [56]). For standard torque parameters and $t_{0}=3 \mathrm{Myr}(5 \mathrm{Myr})-$ consistent with observed disk timescales (Hernández et al. 2007) - the survival fraction has values $p_{S} \approx 0.16(0.10)$. The Type I torque parameter also depends on the mass of the planetary core, which grows with time. The inward migration torques are thus smaller than average at early times and larger at later times. When this time dependence is included, the net survival probability is increased by a modest amount (see Figure 11), with typical values $p_{S}=0.1-0.2$.

For completeness, we have developed an alternate description of the dynamics using an iterative map formalism (given in the Appendix). Although this treatment gives the same results as the Fokker-Planck equation for the same input physics, an iterative map can be useful in several ways: The mapping provides another way to derive - and hence understand - the way in which a finite disk edge enforces an exponentially decreasing survival probability (Section A.3). The Fokker-Planck treatment is limited to small diffusion steps, whereas the iterative map can accommodate large fluctuations. Since boundary conditions are implemented in different ways in the two treatments, some boundary conditions are easier to model with the mapping approach. Finally, the iterative map can easily be generalized to include eccentricity variations and other complications. 
While the iterative map approach is flexible and instructive, it is very computationally intensive: To obtain each of the distributions shown in Figure 12, we needed to perform 100,000 random-walk experiments. This large number is required, in part, because the survival rate is low. In any case, this finding underscores the necessity of using complementary methods such as the Fokker-Planck equation.

\subsection{Discussion}

One of the interesting results of this study is the complicated nature of the Type I migration epoch. In particular, the formation and survival of planetary cores involves a series of compromises: [A] In disks with typical properties, Type I torques dominate in the inner disks where $r<10$ AU, and stochastic torques dominate in the outer disk $(r>10 \mathrm{AU})$. The planetary cores are most easily formed just outside the snow-line, near $5 \mathrm{AU}$ for solar-type stars and typical disks; core formation at larger radii is increasingly difficult (as $r$ increases) due to the slower orbit time. As shown here, however, the survival of these cores is enhanced if they start migration at larger radii (Figure 51). [B] Next we find that although turbulence allows planetary cores to survive in spite of Type I migration (Figures 1, 2, and 4), the survival fraction decreases if the diffusion constant becomes too large (Figure 6). [C] We also find that decreasing the disk surface density with time allows for more planetary cores to survive (Figures 10] and 11); if the surface density decreases too quickly, however, the disk will not have enough gas left to make giant planets. [D] Similarly, the surviving cores are most likely to reside in the outer disk, near $\sim 30$ AU (Figure 9); however, planet formation proceeds much more slowly at large radii and the outer portion of the disk is most susceptible to mass loss through photoevaporation (e.g., Adams et al. 2004). Because of these compromises, the survival of planetary cores depends on the interplay between a large number of ingredients, and the Type I migration epoch results in a wide distribution of possible outcomes. These complications, in turn, imply that the resulting planetary systems will display a great deal of diversity.

Although this paper generalizes previous work, a number of additional issues remain to be addressed. We first note that the parameter space for studying the Type I migration problem is huge: In addition to the magnitude of the torque parameters, and their variations with radius, the time dependence of the disk surface density and the planets also play an important role. Next, the true nature of turbulence in circumstellar remains under study, so that its effects on planet migration could vary from system to system and could otherwise alter the assumptions used herein. One important issue is that the numerical simulations that predict turbulence are not fully converged, so that changes in numerical predictions are possible (e.g., Fromang \& Papaloizou 2007). The formulation presented here separates the Type I migration torque from the stochastic turbulent torques and "derives" their amplitudes independently. In practice, however, the presence of turbulence is likely to affect the structure of the disk near the forming planet and can thus alter the Type I torques (e.g., Papaloizou et al. 2007). Fortunately, our formulation of the migration

problem is sufficiently general to address these issues. If, for example, turbulence alters the size of 
the Type I migration torque, or even if it produces a net torque with nonzero mean, this effect can be incorporated by using the proper value of $\gamma$. Another unresolved issue is the correlation time of the turbulence (taken here to be one orbit time). This issue affects the value of the diffusion constant $\beta$.

Another issue that affects the survival of planetary cores is the possible presence of "dead zones", i.e., regions in the disk where MRI is not active because of insufficient ionization (Gammie 1996). In these zones, turbulence is absent and hence the diffusion constant vanishes. Since Type I torques continue to operate in these regions, planetary cores migrate inward and can be lost. Although the structure and radial extent of dead zones in disks are not fully understood, these zones are expected in the annulus from about 0.3 to $3 \mathrm{AU}$. In the extreme case, the outer edge of the dead zone $(\sim 3 \mathrm{AU})$ would provide the effective inner boundary for the diffusion problem addressed in this paper - all planets that reach this location would quickly be swept inward by Type I torques and eventually accreted by the star. However, this picture contains many complications: The outer (top/bottom) layers of the disk remain ionized, and hence turbulent. These regions provide some (highly reduced) torques (Oishi et al. 2007), and allow for turbulent mixing that can enliven the dead zones (Ilgner \& Nelson 2008, Inutsuka \& Takayoshi 2005). In addition, the lower viscosity in the dead zone can allow the planet to open a gap at lower masses and thereby reduce its inward speed (e.g., Matsumura et al. 2007). These issues render the migration scenario complex, and should be addressed in future work.

Finally, we note that this paper only addresses the survival of planetary cores. Many additional steps are required to produce fully formed giant planets. After the planetary cores reach a sufficiently large mass $\left(30-100 M_{\oplus}\right)$, they clear gaps in the disks and migrate more slowly. This study shows that the planetary cores that survive the embedded phase of migration will reside in the outer disk (Figure 9). If the planets did not migrate after clearing gaps, the results of this model would predict many more giant planets in wide orbits $(a=10-30 \mathrm{AU})$ compared with those in close orbits $(a \approx 0.1 \mathrm{AU})$; the outer planets would be more abundant by a factor of 6 to 100, depending on the values of the torque parameters (see Figure 9). However, the surviving cores will move inward through Type II migration as they continue to grow. This later migration phase is not calculated herein, but it will act to move the distribution of semi-major axes inward, and should be considered in future work.

The basic issue addressed in this paper is that Type I migration tends to move planetary cores inward too rapidly, before they can clear gaps and before they can grow into giant planets. Building on previous work (LSA, NP, JGM), we have explored a solution to this Type I migration problem where the planetary cores experience a random walk due to turbulent perturbations. Although this solution is successful in many ways, other physical processes can contribute. If the planetary orbit is eccentric, for example, the Type I torques are weaker (Papaloizou \& Larwood 2000); migration can thus be slowed down if some process can maintain orbital eccentricities. Similarly, the torques are weaker if the disk itself maintains global (non-axisymmetric) distortions (Papaloizou 2002). Another contributing factor is the detailed structure of the disk, which can depart from the power- 
law forms considered here. Opacity transitions affect the disk structure and hence the migration rates (Menou \& Goodman 2004); for sufficiently high opacities, the migration can even be directed outwards (Paardekooper \& Mellema 2006). Strong magnetic fields can dominate over Type I torques (Terquem 2003), moving planets both inward and outward. Finally, the inner disk can be truncated by magnetic effects (Shu et al. 2007) so that planetary cores cannot migrate all the way to the stellar surface. In closing, the challenge left for the future is to determine how all of these processes — and others - work together to extend the time required for Type I migration and thereby allow giant planets to form.

We thank Nuria Calvet, Greg Laughlin, and Daniel Lecoanet for useful discussions. This work was supported in part by the Michigan Center for Theoretical Physics. FCA is supported by NASA through the Origins of Solar Systems Program via grant NNX07AP17G. AMB is supported by the NSF through grant DMS-604307. In addition, AMB and FCA are jointly supported by Grant Number DMS-0806756 from the NSF Division of Applied Mathematics.

\section{A. AN ITERATIVE MAPPING SCHEME FOR MIGRATION}

As an alternative to the Fokker-Planck treatment presented in the text, this Appendix develops an simple iterative mapping approach. The evolution of planetary angular momentum evolution can be described by an iterative map that includes both the Type I inward migration and the stochastic changes due to turbulent forcing. The map can thus be written in the form

$$
j_{k+1}=\left[1-\left(\frac{\Delta j}{j}\right)_{1}\right]_{k}\left[1+\left(\frac{\Delta j}{j}\right)_{T}\right]_{k} j_{k},
$$

where the subscript labels the step number. Note that the angular momentum increments occur over the time scale $\tau_{T}$ on which the turbulent fluctuations are independent (roughly an orbit time). As a result, we must include a second map to track the time, i.e.,

$$
t_{k+1}=t_{k}+\left(\tau_{T}\right)_{k}=t_{k}+\frac{2 \pi f_{\alpha}}{\Omega_{k}} .
$$

The full map can be written in terms of an ordered product. The angular momentum at orbit number $N$ becomes

$$
j_{N}=j_{0} \prod_{k=1}^{N}\left[1-\left(\frac{\Delta j}{j}\right)_{1}\right]_{k}\left[1+\left(\frac{\Delta j}{j}\right)_{T}\right]_{k},
$$

where $j_{0}$ is the starting value. Note that the factors, in general, depend on angular momentum and are evaluated at the previous step. As a result, the order of the product matters. Although the random parameter $\xi$ that determines the realization of the turbulent torque is independently distributed, the angular momentum increments due to turbulence are not. 


\section{A.1. An Aside on Mapping Approximations}

In the treatment given above, we made the approximation such that the angular momentum increments are small per orbit. In particular, we have multiplied the torque by the orbit time scale $2 \pi f_{\alpha} / \Omega$ instead of integrating over the same time interval. For power-law disks, one can easily perform the integration and obtain more accurate formulae. In practical terms, however, the uncertainties in the turbulent forcing are larger than the accuracy gained. In order to understand the relationship between the iterative map and the Fokker-Planck treatments, however, we need the integrated result. For the benchmark case where $[(\Delta j) / j]$ is constant, we thus obtain

$$
d j=-T_{1} d t=-\left[T_{1}\right]_{k}\left(\frac{j}{j_{k}}\right)^{-2} \frac{2 \pi}{\Omega} d n=-\left[T_{1}\right]_{k} \frac{2 \pi}{\Omega_{k}} \frac{j}{j_{k}} d n
$$

where the subscript $k$ denotes that the quantities are to be evaluated at the beginning of the $k t h$ time interval. Here, $d n$ is the increment of the number of orbits, so we need to integrate from $n=0$

to $n=f_{\alpha}$. After integrating, the change in angular momentum over the time scale $\tau_{T k}$ (for the current radial location) takes the form

$$
1-\left(\frac{\Delta j}{j}\right)_{1}=\exp \left\{-\frac{\left[T_{1}\right]_{k}}{j_{k}} \tau_{T k}\right\}
$$

Note that the product of many such factors takes the form

$$
\Pi^{(N)}=\prod_{k=1}^{N}\left[1-\left(\frac{\Delta j}{j}\right)_{1}\right]_{k}=\prod_{k=1}^{N} \exp \left\{-\frac{\left[T_{1}\right]_{k}}{j_{k}} \tau_{T k}\right\}=\exp \left\{-\sum_{k=1}^{N} \frac{\left[T_{1}\right]_{k} \tau_{T k}}{j_{k}}\right\} .
$$

For this case, $\left[T_{1}\right] \propto j^{-2}$ and $\tau_{T} \propto \Omega^{-1} \propto j^{3}$ so that $\left[T_{1}\right] \tau_{T} / j$ is a constant (the same for each cycle). As a result, we can write the product in the form

$$
\Pi^{(N)}=\exp \left\{-\sum_{k=1}^{N} \frac{\left[T_{1}\right]_{0} \tau_{T k}}{j_{0}}\right\}=\exp \left\{-\frac{\left[T_{1}\right]_{0} \tau_{T k}}{j_{0}} N\right\}=\left[\exp \left\{-\frac{\left[T_{1}\right]_{0} \tau_{T k}}{j_{0}}\right\}\right]^{N},
$$

where the subscript zero denotes that the quantities are to be evaluated at the beginning of the migration epoch $(t=0)$.

\section{A.2. Example}

As a working example, we consider the standard disk where $q=1 / 2$ and $p=3 / 2$. In this case, the relative angular momentum changes due to Type I migration are independent of $j$, i.e., the planet loses a fixed fraction of angular momentum per orbit (or per time interval $\tau_{T}$ ). The effect of Type I migration on the planet is just a constant factor $F_{1}$ in the iterative map. Here we take

$$
\left(\frac{\Delta j}{j}\right)_{1}=10^{-5} \quad \Rightarrow \quad F_{1}=\exp \left[-10^{-5}\right] .
$$


For this same disk model, the relative angular momentum perturbations due to turbulent fluctuations are linear in $j$, and the iterative map takes the form

$$
j_{n+1}=F_{1}\left[1+A \xi\left(j_{n} / j_{0}\right)\right]_{n} j_{n}
$$

where $\xi$ is a random variable and $A$ sets the amplitude. Here we take $\xi$ to follow a gaussian distribution with zero mean and unit variance. The amplitude is set to $A=10^{-3}$, which corresponds to our standard value $\beta=1$ in the Fokker-Planck equation (see Section 4). The scale $j_{0}$ is the angular momentum for a circular orbit at $a=1 \mathrm{AU}$ around a solar type star $\left(M_{*}=1.0 M_{\odot}\right)$.

The starting radii are taken to be distributed in a narrow gaussian centered on $x=\sqrt{30}$, corresponding to the angular momentum appropriate for a circular orbit at $r=30 \mathrm{AU}$. The resulting distributions of radial locations are shown in Figure 12 for times $t=1,3$, and 5 Myr. These distributions are both qualitatively and quantitatively like those produced by solutions to the Fokker-Planck equation (see the main text). Compared to the Fokker-Planck solutions, these distributions have a slightly smaller height near the outer boundary. This discrepancy is due to the different ways in which the outer boundary condition is implemented in the two methods. In the Fokker-Planck treatment, we use a standard zero-flux condition at the disk edge. For this iterative map approach, the migrating planet is not allowed to cross the radius corresponding to the disk edge. This latter boundary condition is thus implemented in a "one planet at a time" manner and does not exactly produce the smooth (zero-derivative) solutions of the Fokker-Planck equation.

\section{A.3. Heuristic Argument for Exponential Decay}

We can use this iterative map formalism to show that the number of surviving planetary cores is a decaying exponential function. This argument applies when the disk has a well-defined outer edge.

We consider only late times, when most planets would be swept into the star by Type I migration in the absence of diffusion. In this regime, most of the surviving planets will be piled up in the vicinity of the outer disk edge. The change in angular momentum due to turbulent torques, which in general depend on the planet's location, can be simplified by evaluating the torque amplitude at a constant value near the disk edge. The change in angular momentum due to Type I migration is already (in the standard case) a constant. As a result, the net effect of one cycle of the iterative map is to change the angular momentum by the factor

$$
\mathcal{F}=\mathcal{F}_{1} \mathcal{F}_{T}=\left[1-\left(\frac{\Delta j}{j}\right)_{1}\right]\left[1+\left(\frac{\Delta j}{j}\right)_{T} \xi\right],
$$

where $\xi$ is a random variable of zero mean and unit variance, and the other factors are now constant. After $N$ iterations, the accumulated angular momentum can be written as the product

$$
j_{(N)}=j_{0} \mathcal{F}_{1}^{N} \prod_{k=1}^{N}\left[1+\left(\frac{\Delta j}{j}\right)_{T} \xi_{k}\right],
$$




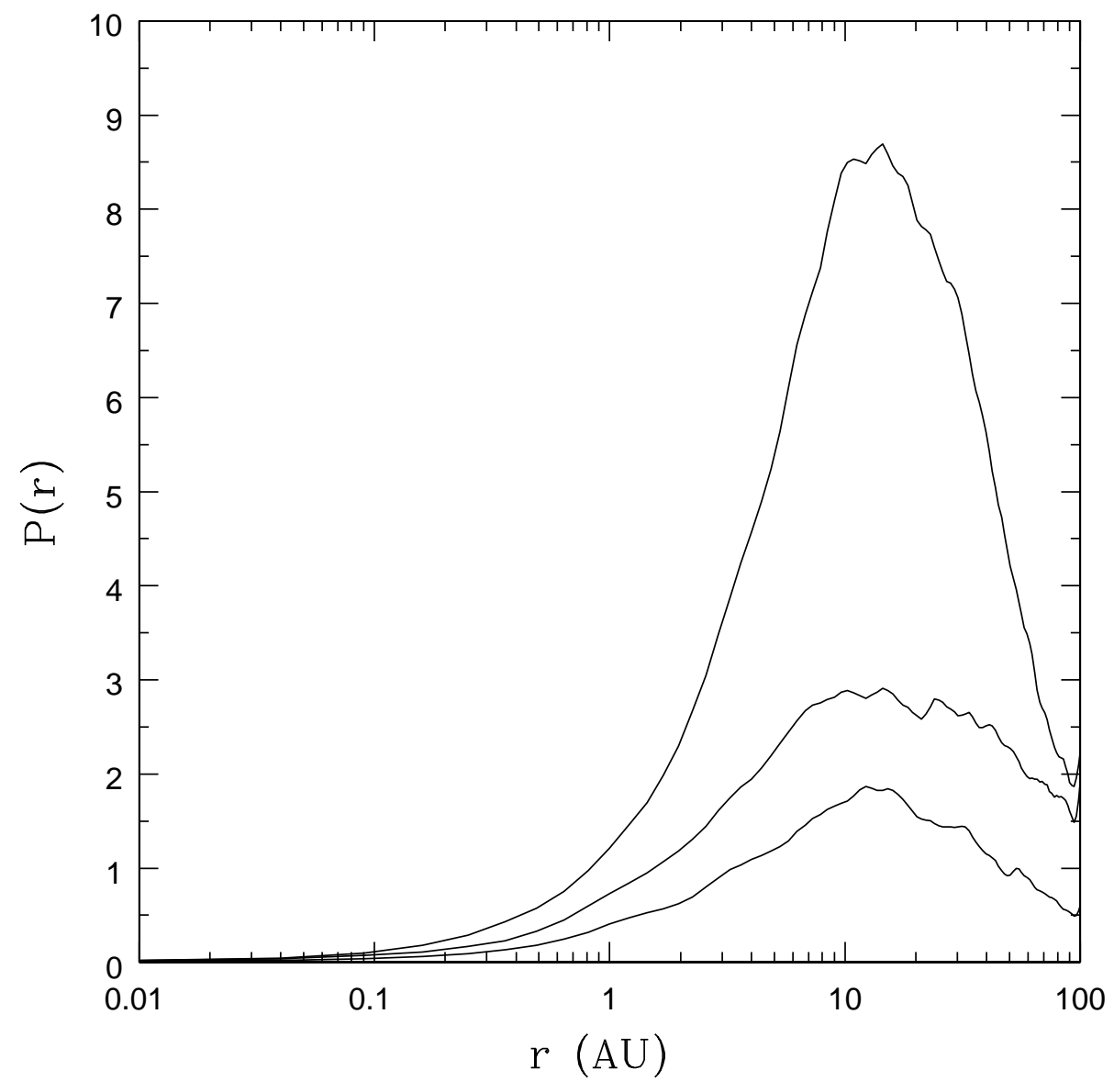

Fig. 12.- Distributions of migrating planetary cores at three times: $1 \mathrm{Myr}, 3 \mathrm{Myr}$, and $5 \mathrm{My}$ (from top to bottom). These results provide an example of the iterative mapping scheme developed in this Appendix. Here, the initial condition is taken to be a narrow gaussian distribution centered on $x=\sqrt{30}$, i.e., the angular momentum appropriate for a circular orbit at radius $r=30$ AU. 
which can be rewritten in the more convenient form

$$
\ln \left[j_{(N)} / j_{0}\right]=N \ln \mathcal{F}_{1}+\sum_{k=1}^{N} \ln \left[1+\left(\frac{\Delta j}{j}\right)_{T} \xi_{k}\right] .
$$

Working to leading order, we simplify the sum so that the angular momentum variable takes the form

$$
\ln \left[j_{(N)} / j_{0}\right]=N \ln \mathcal{F}_{1}+\left(\frac{\Delta j}{j}\right)_{T} \sum_{k=1}^{N} \xi_{k},
$$

which is correct to the same order as the Fokker-Planck treatment (see below). The final sum in equation (A13) is the sum of random variables. In the limit of large $N$, the long time limit, the composite variable $\zeta_{N}=\sum \xi_{k}$ will have a distribution that approaches a normal form (due to the Central Limit Theorem, e.g., Richtmyer 1978). Further, since the individual variables $\xi_{k}$ have unit variance, the composite variable $\zeta_{N}$ has variance $\sigma_{\zeta}^{2}=N$.

Survival of the planetary core requires that the angular momentum remain larger than that of the star, i.e.,

$$
j_{(N)}>j_{*} \equiv j_{0} \mathcal{F}_{1}^{K}
$$

where the second equality defines $K$, the number of steps required for Type I migration to reduce the angular momentum of the starting state $j_{0}$ to that of the stellar surface $j_{*}$. Combining the above results implies the following requirement for planetary survival

$$
\zeta_{N}>\frac{(N-K) \ln \mathcal{F}_{1}^{-1}}{(\Delta j / j)_{T}} \approx(N-K) \frac{(\Delta j / j)_{1}}{(\Delta j / j)_{T}} \equiv \zeta_{*} .
$$

The probability of planetary survival $p_{S}$ is thus given by the integral

$$
p_{S}(N)=A \int_{\zeta_{*}}^{\infty} \exp \left[-\zeta^{2} / 2 N\right] d \zeta,
$$

where $A$ is a normalization constant. Note that the planetary cores do not necessarily have a gaussian distribution in their initial state, so that the constant $A$ can be less than that corresponding to the standard normalization at $t=0$. In the regime of interest, at late times when $N$ is large, the integral in equation (A16) can be evaluated asymptotically to obtain

$$
p_{S}(N)=A \frac{N}{\zeta_{*}} \exp \left[-\zeta_{*}^{2} / 2 N\right]\left[1-\frac{N}{\zeta_{*}^{2}}+\frac{3 N^{2}}{\zeta_{*}^{4}}+\ldots\right] .
$$

In the extreme limit $N \gg K$, the survival probability can be written in the form

$$
p_{S}(N)=\frac{A}{\mathcal{R}} \exp \left[-\left(\mathcal{R}^{2} / 2\right) N\right]
$$

where $\mathcal{R} \equiv(\Delta j / j)_{1} /(\Delta j / j)_{T}$. Since Type I migration dominates in the inner disk, but the torques increase their amplitude relative to Type I torques as the radius increases, we expect the ratio $\mathcal{R}$ to 
be order unity in the outer disk. In addition, since the parameter $N$ counts orbits, but the orbits in question are those near the outer disk edge, the parameter $N$ is proportional to time. Thus, the above result shows that the survival probability decays exponentially with time.

The decay rate is overestimated in the above analysis because we have taken the limit $N \gg K$. In practice, orbits will decay due to Type I torques in $K \sim 10^{5}$ orbits, typically a few Myr, so that $N$

will be comparable to (but still larger than) $K$. This correction does not change the result that the fraction of surviving planets decays exponentially, but it does lower the decay rate. Operationally, $N$ should be replaced by $(N-K)^{2} / N$ in the argument of the exponential in equation (A18).

\section{REFERENCES}

Abramowitz, M., \& Stegun, I. A. 1970, Handbook of Mathematical Functions (New York: Dover) $(\mathrm{AS})$

Adams, F. C., Hollenbach, D., Laughlin, G., \& Gorti, U. 2004, ApJ, 611, 360

Adams, F. C., Laughlin, G., \& Bloch, A. M. 2008, ApJ, 683, 1117

Adams, F. C., \& Shu, F. H. 1986, ApJ, 308, 836

Cassen, P., \& Moosman, A. 1981, Icarus, 48, 353

Chiang, E. I., \& Goldreich, P. 1997, ApJ, 490, 368

Fromang, S., \& Papaloizou, J.C.P. 2007, A\&A, 476, 1113

Gammie, C. F. 1996, ApJ, 457, 355

Goldreich, P., \& Tremaine, S. 1979, ApJ, 233, 857

Goldreich, P., \& Tremaine, S. 1980, ApJ, 241, 424

Haisch, K. E., Lada, E. A., \& Lada, C. J. 2001, ApJ, 553, L153

Hernández, J., Hartmann, L., Megeath, T., Gutermuth, R., Muzerolle, J., Calvet, N., Vivas, A. K., Briceo, C., Allen, L., Stauffer, J., Young, E., \& Fazio, G. 2007, ApJ, 662, 1067

Hillenbrand, L. A. 2008, Phys. Scrip., T130, 014024

Ilgner, M., \& Nelson, R. P. 2008 A\&A, 483, 815

Inutsuka, S., \& Sano, T. 2005, ApJ, 628

Johnson, E. T., Goodman, J., \& Menou, K. 2006, ApJ, 647, 14 (JGM)

Kuchner, M. J. 2004, ApJ, 612, 1147 
Laughlin, G., Steinacker, A., \& Adams, F. C. 2004, ApJ, 608, 489 (LSA)

Lissauer, J. J., \& Stevenson, D. J. 2007, in Protostars and Planets V, eds. B. Reipurth, D. Jewitt, and K. Keil (Tuscon: Univ. Arizona Press), p. 591

Matsumura, S., Pudritz, R. E., \& Thommes, E. W. 2007, ApJ, 660, 1609

Menou, K., \& Goodman, J. 2004, ApJ, 606, 520

Nelson, R. P. 2005, A\&A, 443, 1067

Nelson, R. P., \& Papaloizou, J.C.B. 2004, MNRAS, 350, 849 (NP)

Oishi, J. S., Mac Low, M.-M., \& Menou, K. 2007, ApJ, 670, 805

Paardekooper, S.-J., \& Mellema, G. 2006, A\&A, 459, 17

Papaloizou, J.C.P. 2002, A\&A, 388, 615

Papaloizou, J.C.P., \& Larwood, J. D. 2000, MNRAS, 315, 823

Papaloizou, J.C.B., Nelson, R. P., Kley, W., Masset, F. S., \& Artymowicz, P. 2007, in Protostars and Planets V, eds. B. Reipurth, D. Jewitt, K. Keil (Tuscon: Univ. Arizona Press), p. 655

Papaloizou, J.C.B., \& Terquem, C. 2006, Rep. Prog. Phys., 69, 119

Press, W. H., Teukolsky, S. A., Vetterling, W. T., \& Flannery, B. P. 1992, Numerical Recipes in FORTRAN: The Art of Scientific Computing (Cambridge: Cambridge Univ. Press)

Pringle, J. E. 1981, ARA\&A, 19, 137

Richtmyer, R. D. 1978, Principles of Advanced Mathematical Physics (New York: Springer)

Risken, H. 1984, The Fokker-Planck Equation: Methods of Solution (New York: Springer)

Shu, F. H., Galli, G., Lizano, S., Glassgold, A. E., \& Diamond, P. H. 2007, ApJ, 665, 535

Tanaka, H., Takeuchi, T., \& Ward, W. R. 2002, ApJ, 565, 1257

Terquem, C. 2003, MNRAS, 341, 1157

Udry, S., Fischer, D., \& Queloz, D. 2007, in Protostars and Planets V, eds. B. Reipurth, D. Jewitt, and K. Keil (Tuscon: Univ. Arizona Press), p. 685

Ward, W. R. 1997a, Icarus, 126, 261

Ward, W. R. 1997b, ApJ, 482, L211

Weidenschilling, S. J. 1977, Ap\&SS, 51, 153 